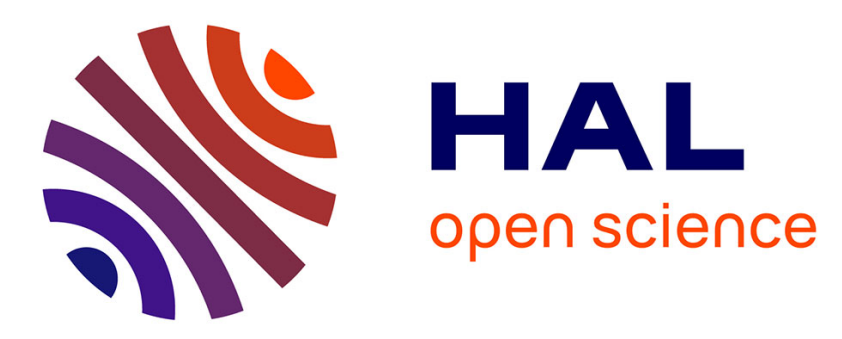

\title{
Géoarchéologies des contextes urbains : mieux comprendre les modalités de l'artificialisation des géosystèmes
}

\author{
Quentin Borderie, Rowena Banerjea, Stéphane Bonnet, Yannick Devos, \\ Cristiano Nicosia, Christophe Petit, Ferréol Salomon, Nathalie Schneider, \\ Barbora Wouters, Patrice Wuscher
}

\section{To cite this version:}

Quentin Borderie, Rowena Banerjea, Stéphane Bonnet, Yannick Devos, Cristiano Nicosia, et al.. Géoarchéologies des contextes urbains: mieux comprendre les modalités de l'artificialisation des géosystèmes. Archimède: archéologie et histoire ancienne, 2020, 7, pp.141-157. 10.47245/archimede.0007.act.04 . halshs-02893402

\section{HAL Id: halshs-02893402 \\ https://shs.hal.science/halshs-02893402}

Submitted on 8 Jul 2020

HAL is a multi-disciplinary open access archive for the deposit and dissemination of scientific research documents, whether they are published or not. The documents may come from teaching and research institutions in France or abroad, or from public or private research centers.
L'archive ouverte pluridisciplinaire HAL, est destinée au dépôt et à la diffusion de documents scientifiques de niveau recherche, publiés ou non, émanant des établissements d'enseignement et de recherche français ou étrangers, des laboratoires publics ou privés. 


\section{ARCHIMÈDE N N 7}

\section{DOSSIER THÉMATIQUE : GESTES RITUELS. DE LA TRACE À L'INTERPRÉTATION}

\section{ACTUALITÉ DE LA RECHERCHE GÉOSCIENCES ET ARCHÉOLOGIE : INTERACTIONS, COMPLÉMENTARITÉS ET PERSPECTIVES}

114 Bruno GAVAZZI

Heureuses rencontres. Vers le développement d'approches intégrées en géosciences et archéologie

119 Jean-Paul BRAVARD

Dialogue interdisciplinaire : de I'unité stratigraphique aux interactions culture-environnement

129 Ferréol SALOMON

Les origines d'Ostie : quelles interactions avec la dynamique d'embouchure? (Delta du Tibre, Italie)

141 Quentin BORDERIE, Rowena Y. BANERJEA, Stéphane BONNET, Yannick DEVOS, Cristiano NICOSIA, Christophe PETIT, Ferréol SALOMON, Nathalie SCHNEIDER, Barbora WOUTERS, \& Patrice WUSCHER Géoarchéologies des contextes urbains : mieux comprendre les modalités de l'artificialisation des géosystèmes

158 Patrice WUSCHER, Christophe JORDA, Quentin BORDERIE, Nathalie SCHNEIDER \& Laurent BRUXELLES De la formation géologique à la tranchée : trouver et comprendre les sites archéologiques menacés par les travaux d'aménagement du territoire

176 Morgan MILLET \& Michel GUÉLAT

Les vestiges antiques de Rennaz-Noville (Vaud, Suisse) et leur contexte sédimentaire : nouvelles évidences de l'écroulement du Tauredunum

188 Théophile PIAU, François BÉTARD, Fabienne DUGAST, Gilles ARNAUD-FASSETTA \& Vincent VIEL Dynamique géomorphologique holocène et occupation humaine dans le bassin-versant de l'Eure (Bassin de Paris, France) : potentiels d'une approche géoarchéologique multiscalaire et diachronique

205 Dominique SCHWARTZ, Vincent ROBIN, Pierre ADAM, Philippe SCHAEFFER, Anne GEBHARDT, Pierre-Alexis HERRAULT, Benjamin KELLER, Daniele DAPIAGGI, Claire STEVENEL, Maxime THISS, Martine TRAUTMANN \& Damien ERTLEN

Les géosciences au service de l'archéologie agraire. Une étude de cas sur les rideaux de culture de Goldbach (68)

217 Étienne MANTEL, Stéphane DUBOIS, Jonas PARÉTIAS, Victor VISQUESNEL-SCHLOSSER, Corentin VOISIN, Bruno GAVAZZI \& Matthieu RICHARD

Étudier I'occupation d'une ville : les enjeux du PCR « Topographie générale et insertion territoriale de I'agglomération antique de Briga »

231 Charlène MOREL

Un modèle pour comprendre l'influence de l'état de la recherche, des processus post-déposition et de l'attractivité sur la découverte des sites archéologiques dans le Kochersberg (Bas-Rhin, Alsace, Grand-Est, France)

241 Lizzie SCHOLTUS

Spatialisation des découvertes, modélisation du passé. L'informatique au service de l'archéologie

254 Guillaume HULIN \& François-Xavier SIMON

Inrap et géophysique : vers une approche raisonnée

260 Hugo REILLER, Matthieu FUCHS, \& Bruno GAVAZZI

Approche multi-méthodes expérimentale pour l'étude d'un site d'occupation romaine et médiévale à Horbourg-Wihr

272 François-Xavier SIMON, Julien GUILLEMOTEAU, Guillaume HULIN, Joachim RIMPOT, Julien THIESSON \& Alain TABBAGH

De nouvelles perspectives pour les applications des méthodes électromagnétiques basse fréquence en archéologie

283 Rémy WASSONG \& Bruno GAVAZZI

Apport des prospections magnétiques haute résolution à la compréhension d'un habitat protohistorique :

l'exemple du site de hauteur fortifié du Maimont 


\title{
GÉOARCHÉOLOGIES DES CONTEXTES URBAINS : MIEUX COMPRENDRE LES MODALITÉS DE L'ARTIFICIALISATION DES GÉOSYSTĖMES
}

\author{
Quentin BORDERIE ${ }^{1,2, *}$, Rowena Y. BANERJEA ${ }^{3}$, Stéphane BONNET ${ }^{4,5}$, Yannick DEVOS 6 , \\ Cristiano NICOSIA ${ }^{7}$, Christophe PETIT ${ }^{2}$, Ferréol SALOMON ${ }^{8}$, Nathalie SCHNEIDER ${ }^{8,9}$, Barbora \\ WOUTERS $^{6}$, Patrice WUSCHER ${ }^{8,10}$ \\ ${ }^{1}$ Conseil départemental d'Eure-et-Loir. ${ }^{2}$ UMR 7041 ArScAn, équipe Archéologies environnementales, \\ Université Paris 1 Panthéon-Sorbonne. ${ }^{3}$ University of Reading, Royaume-Uni. ${ }^{4}$ Ville d'Aix-en-Provence \\ ${ }^{5}$ UMR 7299 Centre Camille Julian. ${ }^{6}$ Maritime Cultures Research Institute (MARI), Vrije Universiteit Brussel \\ (VUB), Belgique. ${ }^{7}$ Dipartimento dei Beni Culturali, Università di Padova, Italie. ${ }^{8}$ UMR 7362 Live, CNRS, \\ Université Strasbourg. ${ }^{9}$ Institut national de recherches archéologiques préventives \\ ${ }^{9}$ Maritime Cultures Research Institute (MARI), Vrije Universiteit Brussel (VUB), Belgique \\ ${ }^{10}$ Archéologie Alsace. * quentinborderie@yahoo.fr
}

\section{RÉSUMÉ}

L'objet des approches géoarchéologiques de l'urbain est constitué du site, de la ville en tant qu'artefact et bassin artificiel de sédimentation anthropique. Dans ces contextes, les relations entre les sociétés et le géosystème produisent des formations pédo-sédimentaires denses et diverses. Les échelles traitées sont celles d'un socio-système complexe, incluant le substrat pédo-géo-chimique local, les formations superficielles artificielles et les relations avec I'hinterland. Dans un contexte actuel d'artificialisation accéléré des espaces, comprendre ces systèmes peut être une clé pour aborder l'Anthropocène. Différentes approches géoarchéologiques de cet objet urbain hybride, conduites en Europe, sont exposées dans cet article. De l'âge du Fer à la période moderne, elles abordent les questions de topographie préurbaine, de gestion

\section{MotS-CLÉs}

Socio-écosystèmes,

ville,

anthropisation,

artificialisation,

pollutions,

pédologie,

micromorphologie,

archéologie,

paysage,

terres noires. sociale des flux de matériaux, de pollutions, d'occupation des espaces et d'évolution des stratifications. Les résultats montrent la diversité des relations entre les sociétés et les sols, dans une co-construction sur le temps long de systèmes urbains artificiels.
Geoarchaeological approaches to ancient urban contexts focus on the study of the town as an artefact, and as an artificial and anthropogenic sedimentary basin. In such context the relationship between societies and the geosystem generates diverse and dense pedo-sedimentary formations. The studied scales of such a complex socio-system encompass local geochemical substrate, artificial superficial formations, and relationship between town and hinterland. In the actual conditions of massive and accelerated artificialisation of spaces, the study of those systems can give new clues to better understand the Anthropocene. Different geoarchaeological approaches developed in Europe to study this hybrid urban object are exposed here. From the Iron Age to modern period, they deal with questions of pre-urban topography, social management of material flows, pollutions, occupation of spaces, stratigraphy, and taphonomy. The obtained results underline the diversity of the relations between societies and soils, rivers, hazards in a long-term co-construction of artificial urban systems, which are our heritage now.

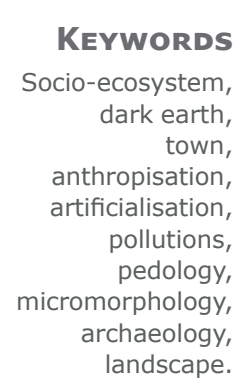




\section{INTRODUCTION}

\section{LA VILLE ET L'URBAIN}

L'impact des modalités anciennes d'urbanisation du paysage et des écosystèmes pourrait paraître bien insignifiant face à l'urbain global, à l'artificialisation effrénée des sols à laquelle nous assistons aujourd'hui et qui contribuent à définir la période actuelle en tant qu'Anthropocène [1]. Pourtant, c'est peut-être cette diversité des formes complexes de l'urbain, depuis l'agglomération néolithique et l'oppidum de l'âge du Fer jusqu'à la cité antique et à la ville médiévale, qui contribue aux « racines de l'Anthropocène » [2] et pose les jalons d'une anthropisation totale des paysages.

En échappant à toute définition disciplinaire, les contextes urbains s'imposent d'emblée par leur complexité, la diversité des points de vue qui $s^{\prime} y$ focalisent [3] et la nécessité de les aborder sans déterminisme quant à leur trajectoire historique [4], à l'aide d'estimateurs et de gradient de distribution qui informent sur le caractère plus ou moins urbain des espaces. Au-delà d'une confrontation entre Nature et Culture [5], entre Société et Milieu [6], la diversité des cas de figure et la singularité des rapports à l'espace et au sol qui se créent dans les contextes urbains invitent plutôt à dépasser le paradigme de l'anthropisation pour côtoyer celui de l'artificialisation, c'est-à-dire de passer d'une vision d'un impact sur le milieu à la construction même du milieu [7].

L'objectif de cet article est de montrer l'apport de différentes approches géoarchéologiques des contextes urbains à la compréhension des géo-systèmes artificiels complexes. Après avoir présenté le contexte d'émergence de la géoarchéologie des contextes urbains, en avoir proposé une définition, ce sont les problématiques liées au bassin sédimentaire urbain en tant que tel, puis aux co-constructions société-milieu qui seront exposées, avec les méthodes pour les aborder, au travers d'exemples actuels et de résultats récents. Enfin, des éléments de réflexions sur une approche à long terme de l'enregistrement et de la conservation de ces contextes seront proposés.

\section{LES APPROCHES GÉOARCHÉOLOGIQUES DES CONTEXTES URBAINS}

Initiée au début des années 1980, au sein d'une archéologie environnementale naissante [8], la géoarchéologie des contextes urbains s'est concrétisée en France par les premières collaborations entre géographes, géologues et archéologues lors de grands chantiers de sauvetage. Les prémices d'une vision géomorphologique furent déjà esquissées par Th. Vacquer dès 1844 , lors des travaux haussmanniens à Paris, notamment par la cartographie à l'échelle de la capitale de terres végétales enfouies. À partir de la fin du XX $X^{e}$ s., ce fut le cas lors des fouilles de La Bourse à Marseille et l'étude du port antique [9], ainsi que des travaux préalables à la construction de la ligne $D$ du métro de Lyon [10]. Ces premières collaborations se sont prolongées lors de tables rondes nationales, telle celle organisée par le Centre national d'archéologie urbaine (Cnau) en 1988 [11]. Du fait peut-être d'un cloisonnement disciplinaire fort entre géologues du Quaternaire et archéologues, ces travaux relevaient d'une géomorphologie appliquée dans la ville, plus que d'une géoarchéologie de l'objet urbain.

Dans le même temps, s'est mis en place aux Proche et Moyen-Orient une autre géoarchéologie, lors de fouilles programmées des accumulations sédimentaires urbaines dénommées «tell ». D'abord par la mise en place de cartographies du phosphore [12], ces paysages anthropiques sont abordés à partir des années

[1] BEAU \& LARRÈre 2018.

[2] MAGNY 2019.

[3] PAQUOT et al. 2000.

[4] GaLINIÉ 2000 ; LePETIT 1996.

[7] BEAu \& LARRÈre 2018.

[8] Butzer 1982 ; Butzer et al. 1983 ; HAll \& KENWARD 1982.

[9] MORHANGE et al. 1996.

[10] BRAVARD et al. 1989.

[5] Descola 2005.

[11] BRAVARD et al. 1988.

[6] MUXART et al. 2003.

[12] DAVIDSON $1973 ; 1976$. 
1980 comme un artéfact en soit [13] dont l'étude mobilise les méthodes des sciences de la Terre et du sol [14]. Ces méthodes sont appliquées en France pour l'étude du site de Lattes (Hérault), où le concept d'unités morpho-fonctionnelles est développé [15]. Malheureusement, cette application des méthodes des géosciences aux occupations humaines souffre encore d'une vision tronquée de l'objet d'étude, qui reste limité à une dichotomie et une opposition entre nature et culture, où le degré « d'anthropisation » est cherché dans les tentatives de généralisation [16]. Et I'on reste finalement ici dans une stratigraphie et une pédo-sédimentologie des contextes urbains.

Quant à I'approche pédologique des sols urbains, elle est initiée en France avec le Groupement d'intérêt scientifique (GIS) « sol urbain », dans les années 1990. Quinze laboratoires firent partie de ce GIS, qui fut relayée par le Cnau [17] et dont l'axe 4.1, piloté par H. Galinié, est intitulé « sols anciens ». De cette vaste organisation scientifique, aboutiront un congrès en 1995 et deux ouvrages [18]. Dans le Référentiel pédologique français, D. Baize et M.-C. Girard [19] proposent la définition des « anthroposols archéologiques », parmi les autres anthroposols. Si cette invention permet une certaine prise en compte de la profondeur historique des sols artificiels, tout en suggérant a priori une valeur patrimoniale discutable, elle esquive toute la complexité de leur constitution sur le temps long, les renvoie en marge des autres anthroposols alors qu'elle pourrait les contenir tous.

Depuis les années 1980, les publications et les rencontres consacrées aux approches géoarchéologiques des contextes urbains sont restées sporadiques, même si elles commencent aujourd'hui à se multiplier. Ainsi, de 1986 à 2016, seuls 26 articles de la revue «Geoarchaeology: an international journal », sur un total de 867 , sont consacrés à l'étude des contextes urbains, soit seulement 3\%. Un tel constat peut être fait pour d'autres revues spécialisées de géoarchéologie. Depuis les pionnières rencontres du Cnau à Tours en 1988, Paris a accueilli en 2009 une table ronde intitulée « Géoarchéologie de l'urbain », animée par J. Burnouf et J.-P. Bravard [20]. À Bruxelles en 2015 s'est tenu le Congrès international « Congress of Environmental Archaeology in European Cities », faisant la part belle à la géoarchéologie [21]. À Caen en 2016, une session de la Réunion des Sciences de la Terre (RST) a été consacrée aux « Géosystèmes urbains » et, deux ans plus tard à Barcelone en 2018, une session du colloque annuel de l'European Association of Archaeologists (EAA) était exclusivement consacrée à I' «Urban geoarchaeology ».

\section{POUR DÉFINIR LA GÉOARCHÉOLOGIE DES CONTEXTES URBAINS}

Dans ce contexte d'émergence d'un objet d'étude hybride, comment alors définir la géoarchéologie des contextes urbains ? D'abord en s'inscrivant dans la géoarchéologie telle qu'elle est définie par K. W. Butzer au début des années 1980, «l'étude des interactions dynamiques entre les groupes humains et leurs environnements, par l'étude et l'interprétation des sédiments et des paysages physiques » [22]. Puis, en soulevant des questionnements et des problématiques archéologiques liés aux conditions spécifiques de densité et de diversité propres aux espaces urbains [23], et qui contribuent à la constitution d'une connaissance historique, dans une perception systémique des objets de recherche. Enfin, en pratiquant cette étude du sédiment en mobilisant les méthodes des géosciences, dans une permanente interdisciplinarité.

\section{L'URBAIN : UN BASSIN SÉDIMENTAIRE ANTHROPIQUE}

\section{DE LA TOPOGRAPHIE PRÉ-URBAINE AUX FORMATIONS SUPERFICIELLES ANTHROPIQUES}

Une des premières problématiques abordées par la géoarchéologie des contextes urbains est peut-être celle du site d'implantation des villes, qualifié à tort de « naturel ». Il s'agit ici de mieux connaître les conditions initiales et de développement de ces places centrales, en ayant accès au potentiel sédimentaire du site et à la topographie pré-urbaine. Les travaux conduits à Grenoble sont précurseurs [24], car ils ont permis de reconsidérer la part des matériaux alluviaux et celle des accumulations anthropiques dans le
[13] ROSEN 1986.

[14] MatTHEWs 1992 ; MATTHEWs et al. $1995 ; 1997$.

[15] CAMMAS $1994 ; 1999 ; 2003$.

[16] GÉ et al. 1993.

[17] GARMY 2009.

[18] Barles et al., 1999 ; Cheverry \& Gascuel 2009.
[19] BAIZE \& GiRARD 2009.

[20] BORDERIE 2011a.

[21] Devos et al. 2017b.

[22] BUTZER 1982.

[23] LeVY \& LUSSAULT 2003.

[24] Peiry \& FÉougier 1997. 
sous-sol, ces dernières constituant un dôme qui était, jusque-là, interprété comme fluviatile. Ceux effectués plus récemment à Tours et à Paris sont particulièrement poussés, puisqu'ils ont permis d'identifier finement la topographie antérieure au développement de la ville et, notamment, la géométrie des anciennes formes d'origine alluviales [25]. En ce qui concerne I'urbanisation du site à l'époque médiévale notamment, le cumul d'observations géoarchéologiques à Bruxelles permet de constater le passage progressif d'activités agraires et pastorales à des activités très diversifiées, produisant souvent des rejets importants [26]. À l'échelle d'une région comme l'Alsace (fig. 1), si I'on compare les différents sites d'implantation des villes depuis I'Antiquité, à l'aune des nombreuses données acquises par l'archéologie préventive, on constate qu'aucun déterminisme ne peut être établi. Ainsi, à I'époque antique, I'agglomération de Strasbourg se situe sur une terrasse alluviale couverte de lœss du Pléistocène supérieur exondée durant tout I'Holocène. La plaine alluviale n'est occupée que par un camp militaire. Malgré les aléas, I'humidité et les risque d'inondation, la ville médiévale se développera dans la plaine alluviale [27]. Les agglomérations antiques d'Horbourg-Wihr 2016 [28], d'Oedenburg (BiesheimKunheim, France), installées toutes deux en plaine alluviale ont connu un développement différent et posent la question de l'acceptation de certains aléas (inondations) par les sociétés [29]. Au contraire d'Horbourg dont elle est voisine, l'agglomération médiévale de Colmar se développe quant à elle sur un cône alluvial pléistocène exondé à l'Holocène, tout comme la ville médiévale de Sélestat. Les indices d'occupation antique et la ville médiévale de Mulhouse se trouvent quant à elle en zone humide, dans la plaine alluviale de I'Ill [30], mais la cartographie de son sous-sol demande encore à être précisée.

Dépasser la notion de site et de potentiel permet d'aborder celle de dynamique des formations superficielles anthropiques [31]. Il s'agit ici de qualifier les différents types de formations, leur extension et leurs dynamiques, leur place dans le processus

[25] LAURENT 2007 ; LAURENT \& FONDRILLON 2010 ; Noizet et al. 2013.

[26] Devos et al. 2017a ; 2019.

[27] SCHWIEN et al. 1998.

[28] Higelin 2016 ; Roth-Zehner 2018.

[29] OlLiVe et al. 2006.

[30] VUILLEMIN 2016.

[31] LEBRET et al. 1993.

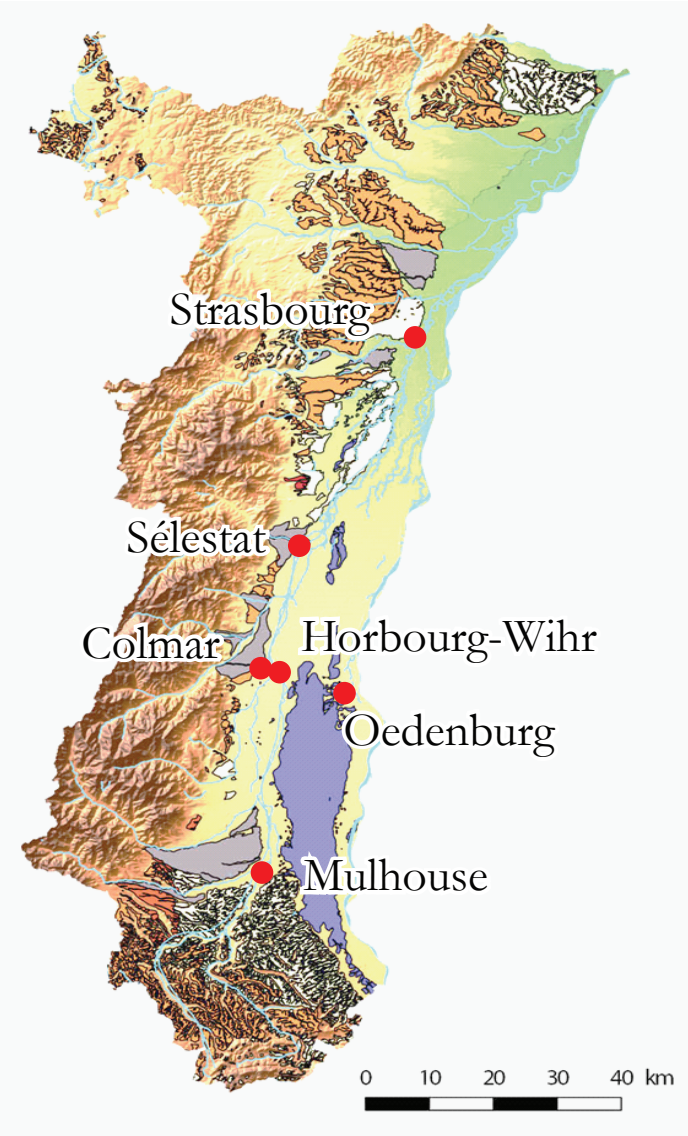

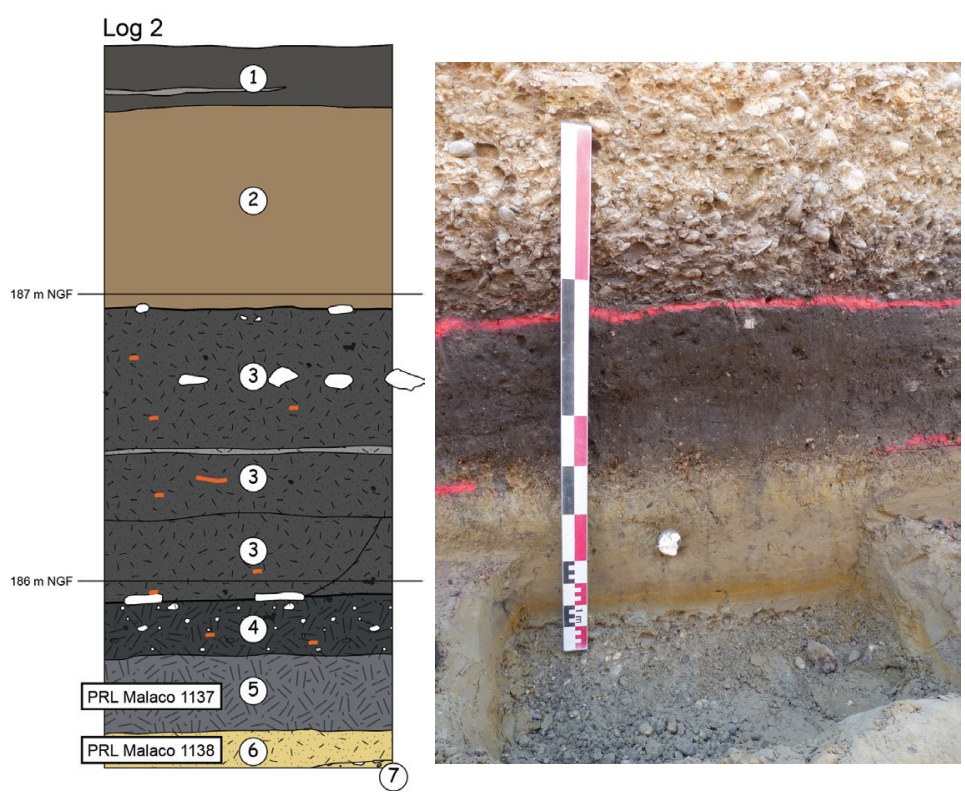

Figure 1 : Localisation des agglomérations alsaciennes citées dans le texte : les zones pléistocènes exondées à l'Holocène sont figurées en jaune (lœss weichsélien), en orange (lœss pléistocènes), en gris (alluvions weichséliennes vosgiennes) et en mauve (alluvions weichséliennes rhénanes). Horbourg-Wihr, log au 50 Grande Rue (Roth-Zehner 2018 ; étude malacologique en cours : Salomé Granai/ LGP Meudon et Géoarchéon ; datations OSL : Frank Preusser/ Université de Freiburg) et vue du profil avec le sol hydromorphe sombre sur lequel prend place l'agglomération antique aux 1 et 3 rue des Écoles. 


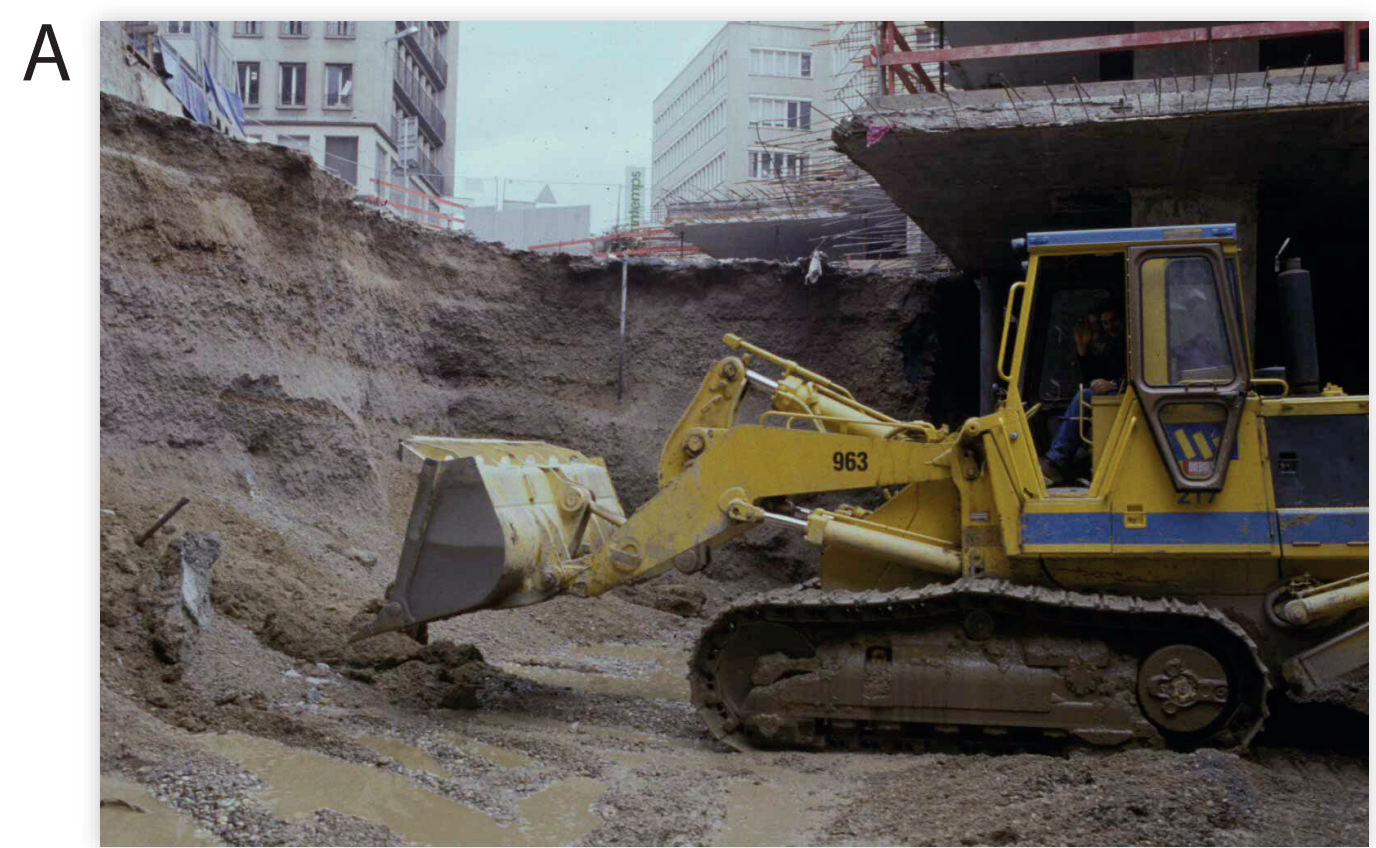

cliché JJ.Schwien

\begin{tabular}{|c|c|c|}
\hline Types de dépôts et évolution & \multicolumn{2}{|l|}{ Datations } \\
\hline$\square$ Pédogenèse au sommet des alluvions & $+700 /+1200 \mathrm{AD}$ & Moyen Âge \\
\hline$\square$ Limon de débordement & $+80 \mathrm{AD}$ & \\
\hline A Limon de débordement & $+480 \mathrm{AD}$ & Antiquitè \\
\hline$\square$ Paléochenal de la Bruche, aménagements de l'Ill & $+250 /+650 \mathrm{AD}$ & \\
\hline Paléochenal de la Bruche, (ENA, rue Kageneck) & $-1200 \mathrm{BC}$ & Âge du Fer \\
\hline $\begin{array}{l}\square \text { Paléosol hydromorphe } \\
\text { Paléochenal sous le fossé des tanneurs }\end{array}$ & $\begin{array}{r}-2600 /-2000 B C \\
-2450 B C\end{array}$ & Âge du Bronze \\
\hline Colluvions loessiques & $-4800 B C$ & Néólithique \\
\hline Gravier mélangé & $-6000 /-5000 \mathrm{BC}$ & \\
\hline Gravier mélangé & $-11000 /-10000 \mathrm{BC}$ & Mésolithique \\
\hline$\square$ Loess weichsélien & & \\
\hline Gravier rhénan non daté & & Quaternaire \\
\hline …. Gravier vosgien non daté & & \\
\hline
\end{tabular}

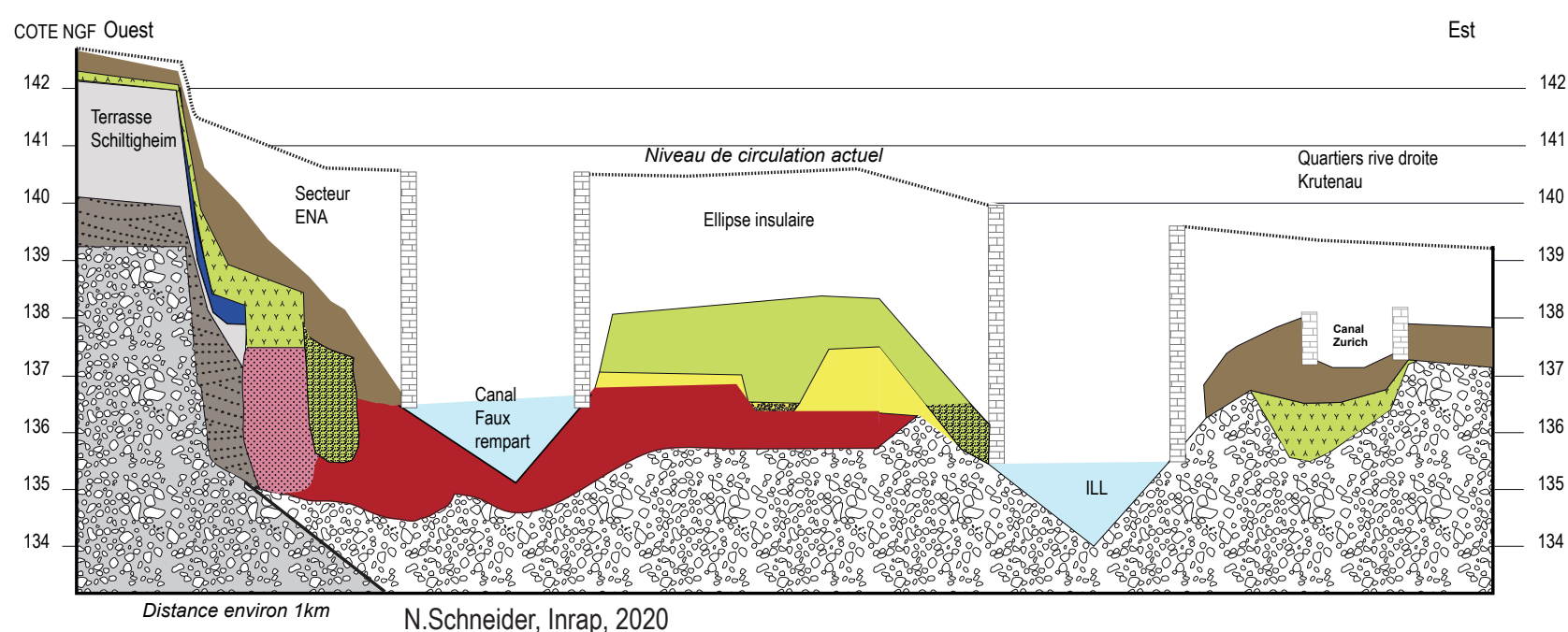

Figure 2

Strasbourg : A : Place de l'Homme de Fer (acquisition de données et DAO J.-J. Schwien, N. Schneider 1991, Tram A) ; B : Chronologie des dépôts alluviaux, synthèse des données 1991-2011, N. Schneider. 
d'artificialisation du bassin sédimentaire. Le récolement et la compilation des données (localisations, unités stratigraphiques, types de sédiments, de sols, chronologies, profondeurs, épaisseur, résultats d'analyses, plans anciens, etc.) sont facilités par l'usage de systèmes d'information géographique (SIG) et permettent de proposer des modèles d'organisation et de dynamique. À Aix-en-Provence, une telle compilation a permis de proposer des cartographies dynamiques des épaisseurs de dépôts holocènes, en lien avec les paléo vallons mais aussi les fossés médiévaux [32]. Ainsi a pu être esquissée la topographie au moment de l'installation d'Aquae Sextiae (Aix-en-Provence). Ces travaux ont conduit à s'interroger sur la nature même du substrat et, in fine, à la découverte de vestiges paléolithiques dans ces formations. Depuis plus de 30 ans, ces récolements sont faits à Strasbourg [33], offrant aujourd'hui une vision dynamique de la constitution du substrat actuel de la ville, qui résulte d'une riche interaction entre les sociétés et l'hydrosystème (fig. 2).

[32] BonNet et al. 2018, 2017, 2010.

[33] SCHWIEN et al. 1998.

[34] ArLAUD et al. 1994; Bini et al., 2018.

[35] Burnouf \& LEVEAU, 2004.

[36] CARCAUD et al. 2019.

\section{DES VILLES EN INTERACTION AVEC LES RÉSEAUX HYDROGRAPHIQUES ET LES LITTORAUX}

En effet, l'une des thématiques les plus abordées est celle de la relation entre les villes et les hydrosystèmes [34]. Identifier les dynamiques de ces socio-écosystèmes [35], les modalités de leur artificialisation et gestion par les sociétés, pour mieux comprendre ce dont nous héritons aujourd'hui et comment intégrer dans nos politiques la gestion de plusieurs siècles d'interactions complexes et de services éco-systémiques rendus (tab. 1). Ces problématiques d'aménagement, de gestion des risques dépassent le cadre du récit historique, pour s'inscrire dans celle du développement durable de demain [36].

À ce titre, l'acquisition de données dans le cadre d'une archéologie préventive, à Strasbourg depuis 1991, parfois dans des conditions exceptionnelles (fig. 2) a conduit à une compréhension fine des modalités de constitution et d'artificialisation du site, depuis la fin du Pléistocène jusqu'aujourd'hui. Malgré la difficulté inhérente à ce type d'environnement urbanisé depuis des siècles, l'hétérogénéité et la discontinuité des données, il a pu être constaté une rupture des apports alluvionnaires grossiers au Tardiglaciaire, une faiblesse récurrente des volumes enregistrés pour le

\begin{tabular}{|c|c|c|}
\hline Thématiques urbaines & Exemples & Références \\
\hline \multirow{6}{*}{$\begin{array}{c}\text { Risques fluviaux } \\
\text { (crues, mobilité fluviale) }\end{array}$} & Lyon, Vienne (France) & Bravard et al. 1989 \\
\hline & Tours (France) & $\begin{array}{c}\text { Arnaud-Fassetta et al. } 2010 \\
\text { Burnouf et al. } 2003\end{array}$ \\
\hline & Arles (France) & Alinne, 2007 \\
\hline & Ostie (Italie) & Salomon et al. 2018 \\
\hline & Oedenburg (France) & Ollive et al. 2006 \\
\hline & Londres (Royaume-Uni) & Cohen \& Wragg, 2017 \\
\hline $\begin{array}{c}\text { Risques littoraux } \\
\text { (tempêtes, mobilité littorale) }\end{array}$ & Dunwich (Royaume-Uni) & Sear et al. 2011 \\
\hline \multirow{4}{*}{ Canaux, chenalisation } & Aquileia (Italie) & Arnaud-Fassetta et al. 2010 \\
\hline & Portus (Italie) & Salomon, 2013 \\
\hline & Bruxelles (Belgique) & Devos et al., 2017c \\
\hline & Gand (Belgique) & Gelaude, 2019 \\
\hline \multirow{2}{*}{ Bassins portuaires } & $\begin{array}{c}\text { Marseille (France), Alexandrie } \\
\text { (Égypte), Tyr (Liban) }\end{array}$ & Marriner et al. 2010 \\
\hline & Bruxelles (Belgique) & Timmermans et al. 2020 \\
\hline Franchissements & Avignon (France) & Vella et al. 2013 \\
\hline
\end{tabular}

Tableau 1

Villes et hydrosystèmes : quelques cas d'étude. 
début de l'Holocène. Les questions liées aux occupations anciennes restent cependant ouvertes : à partir de l'âge du Bronze le site alluvial de Strasbourg est stabilisé, comme l'indique un premier paléosol observé en plusieurs points de l'ellipse insulaire (4 rue Brûlée, Petite Place du Vieux-Marché-Aux-Vins, ENA), au-dessus duquel seules quelques crues ont été enregistrées. La première occupation du site date de l'époque augustéenne. Cette lacune sédimentaire (alluviale et anthropique) ne trouve pas de réponses dans les observations intra muros, mais plutôt dans I'observation des fluctuations du réseau hydrographique dans le fossé rhénan.

\section{DES POLLUTIONS MAL CONNUES ET UN GRADIENT URBAIN-RURAL À MESURER}

Le bassin d'accumulation urbain est caractérisé par une pollution très importante des formations pédo-sédimentaires, c'est-à-dire une augmentation significative des teneurs en éléments (métaux lourds, composés organiques) par rapport au fond pédo-géochimique local. La nature de ces pollutions, leur degré, leur extension et modes de diffusion, ainsi que leur origine sont autant de problématiques encore très peu abordées. Si des travaux pionniers et originaux ont été entrepris outreManche [37], ils restent isolés en France. Pourtant, la mesure de teneurs de métaux lourds pour des contextes antiques ou du début du Moyen Âge est particulièrement révélatrice : $300 \mathrm{mg} / \mathrm{kg}$ dans le port romain à Marseille [38], $1460 \mathrm{mg} / \mathrm{kg}$ à Aix-en-Provence [39], $1800 \mathrm{mg} / \mathrm{kg}$ dans les terres noires du début du Moyen Âge à Metz [40]. Ces taux dépassent très largement les seuils de tolérance actuels. À Bruxelles, on observe des valeurs qui ne dépassent qu'occasionnellement le fond pédo-géochimique local pour les $\mathrm{X}^{\mathrm{e}}$-XIII ${ }^{\mathrm{e}} \mathrm{S}$., tandis qu'à partir du XIVe $s$. des teneurs de métaux lourds deviennent nettement plus élevées [41]. Quant aux éléments constitutifs des composés organiques (carbone, azote, phosphore), des teneurs considérables sont aussi atteintes, avec plus de $20 \mathrm{~g} / \mathrm{kg}$ de phosphore dans les terres noires du début du Moyen Âge à Beauvais, par exemple [42]. Cet enrichissement, aubaine pour la faune du sol, crée des milieux bioturbés où la lecture des unités stratigraphiques devient alors une gageure, dans les terres noires notamment [43].

[37] DAvidson et al. 2006 ; Golding 2008.

[38] Le Roux et al. 2005.

[39] Nin 2002.

[40] Augry \& BORDERIE 2014.

[41] Devos 2019 ; Devos et al. 2019.

[42] BORDERIE, 2012.
Ces pollutions, enrichissement et construction de milieux spécifiquement liés à des pratiques urbaines, donnent une nouvelle limite à notre perception de I'urbain. Ce que Davidson et al. et Golding [44] ont bien montré, c'est qu'il se dessine une différenciation des formations pédo-sédimentaires urbaines par la diffusion des pollutions, repoussant les limites de la ville au-delà d'une zone où est pratiquée une horticulture urbaine, comme à la périphérie de Paris au XIX s. mais aussi dans celle de Metz au Moyen Âge [45]. À Paris, I'analyse des carbonates de spéléothèmes des aqueducs urbains a permis de retracer une histoire de ces pollutions [46]. Les modalités de la diffusion de ces pollutions, atmosphériques ou liées à une redistribution des déchets par les sociétés, demandent encore à être mieux analysées, mais donnent déjà une idée de l'intensité de l'artificialisation des systèmes avant même la première « révolution industrielle».

\section{DES INTERACTIONS SOCIÉTÉS - MILIEU ARTIFICIEL}

\section{LES TRACES PÉDOLOGIQUES DE LA VILLE GAULOISE}

Là où les vestiges monumentaux peuvent faire défaut, comme c'est souvent le cas pour les contextes urbains protohistoriques et de la fin de l'âge du Fer, sauf exception [47], investiguer les traces pédologiques des occupations humaines peut être particulièrement intéressant pour renseigner les activités pratiquées, le degré de fréquentation des lieux. À Chartres, la mise en évidence récurrente de couches de «limons verts », entre le substrat argileux et les occupations antiques a conduit à mettre en place une analyse systématique de ces couches, afin de palier l'impossibilité à les interpréter macroscopiquement. Les premiers résultats montrent que ces limons verts résultent de I'occupation de l'espace à la fin l'âge du Fer par l'accumulation de rejets organiques dans les horizons superficiels du sol, bioturbés [48]. La coloration provient de la présence de nombreux cristaux de vivianite, un phosphate de fer qui se rencontre souvent dans les contextes urbains riches en rejets organiques [49]. Les mêmes caractéristiques se retrouvent à Amboise, dans les dépôts antérieurs à l'occupation de l'âge du Fer de
[43] CAMMAS, 2004 ; MACPHAIL, 1994.

[44] DAVIDSON et al. 2006 Golding 2008.

[45] Nicosia, 2018.

[46] PONS-BRANCHu et al., 2017.

[47] Monin, 2005 ; THION et al., 2003.

[48] BordERIE, 2016A; COUSSOT, 2008.

[49] BORDERIE, 2011B ; MACPHAIL, 1983. 
I'oppidum, ou dans les niveaux laténiens de l'oppidum d'Alésia (observations C. Petit) qui sont pareillement contaminés au phosphore, mais aussi très riches en micro-déchets produits par l'artisanat (agrégats de silice fondue, scories) (fig. 3).

\section{ARTISANAT, COMMERCE, ACTIVITÉS ET ESPACES}

L'artisanat, au même titre que les activités commerciales, donne sens à certaines définitions de l'urbain et en font sa spécificité [50]. Les types d'activités pratiquées en ville, selon les espaces et les pollutions qu'elles génèrent permettent de préciser la connaissance topographique de l'urbain, à l'instar de la gestion des déchets et des structures archéologiques témoins de ces activités. Les travaux récents conduits à Hedeby (Allemagne) ont pu montrer que le travail des métaux précieux (argent) pouvait être pratiqué dans les espaces domestiques, grâce à l'analyse microXRF sur les lames minces de micromorphologie des sédiments des phases précoces de cette place centrale des $\mathrm{VIII}^{\mathrm{e}}-\mathrm{XI}^{\mathrm{e}} \mathrm{s}$. [51]. Pour la période gallo-romaine, des cartographies de concentration en métaux lourds faites dans I'insula IX de Silchester (Royaume-Uni), ont pu être confrontées avec les résultats de l'analyse micromorphologique des sols intérieurs successifs et ainsi, comprendre la complexité de l'organisation des espaces, la localisation des zones de circulation, les modalités de construction de ces sols. Les pollutions au cuivre et au plomb, attribuables à une activité métallurgique, se trouvent dans les espaces domestiques et sont associées à des phases très riches en phosphore et en excréments d'herbivores, interprétables comme des espaces d'écuries [52]. Ces résultats particulièrement précis sont un cas encore isolé de I'application de la micromorphologie aux stratifications de la période romaine.

Les espaces de commerces médiévaux peuvent être difficiles à cerner dans leur matérialité [53]. Si les zones de stockage de denrées peuvent être identifiables à la concentration de certaines structures (comme les silos, tels ceux découverts à Blois [54] pour les $\mathrm{VIII}^{\mathrm{e}-\mathrm{XI}} \mathrm{I}^{\mathrm{e}} \mathrm{s}$.), les espaces d'échanges sont plus difficiles à percevoir. Une approche micromorphologique conduite lors de la fouille des stratifications antérieures au pavement de la place du Grand-Marchéde-Lierre (Belgique) a montré comment s'est opéré, aux $\mathrm{XI}^{\mathrm{e}}$ et $\mathrm{XII}^{\mathrm{e}} \mathrm{s}$., le passage d'un espace cultivé à un espace de commerce qui, jusqu'au XIV ${ }^{e}$ s., recueille les rejets très diversifiés d'un espace occasionnellement très fréquenté [55]. La nature des activités pratiquées dans les espaces urbains peut aussi être

[50] WeBER, 1921 ; WiCKHAM, 2005.

[51] WOUTERS 2016.

[52] BANERJEA 2011 ; COOK et al. $2010 ; 2014$.

[53] BLIECK \& Guiffray 1994.

[54] Aubourg \& JOSSET 2000.

[55] WOUTERS et al. 2017.
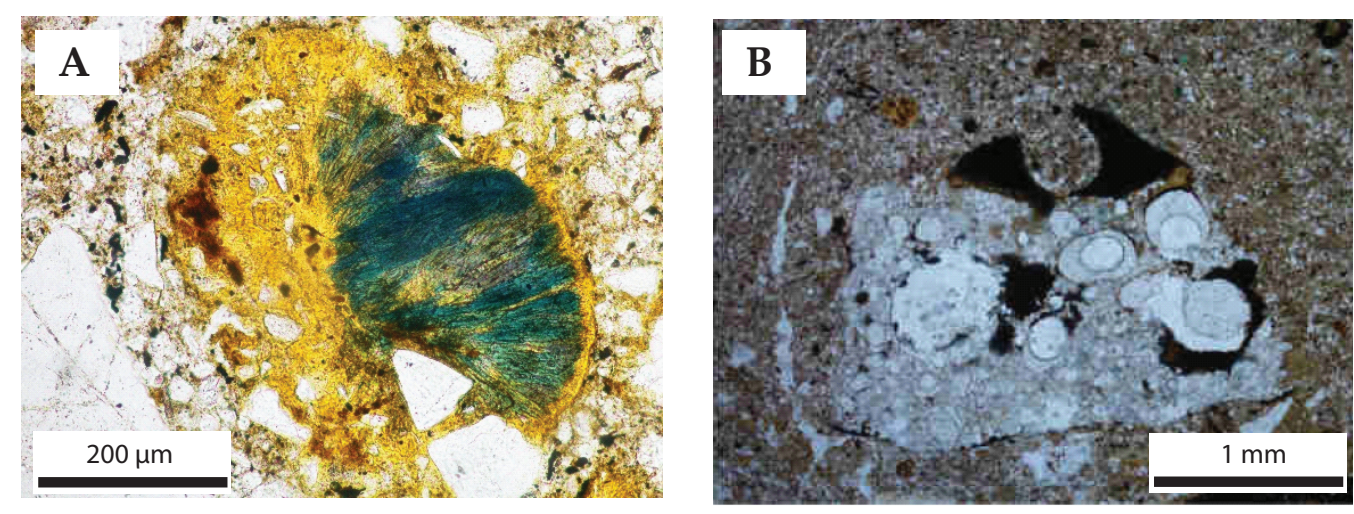

Figure 3

Oppida de Chartres et d'Amboise : pollution des couches archéologiques contemporaines ou antérieures à l'occupation de l'âge du Fer (d'après Borderie 2016a).
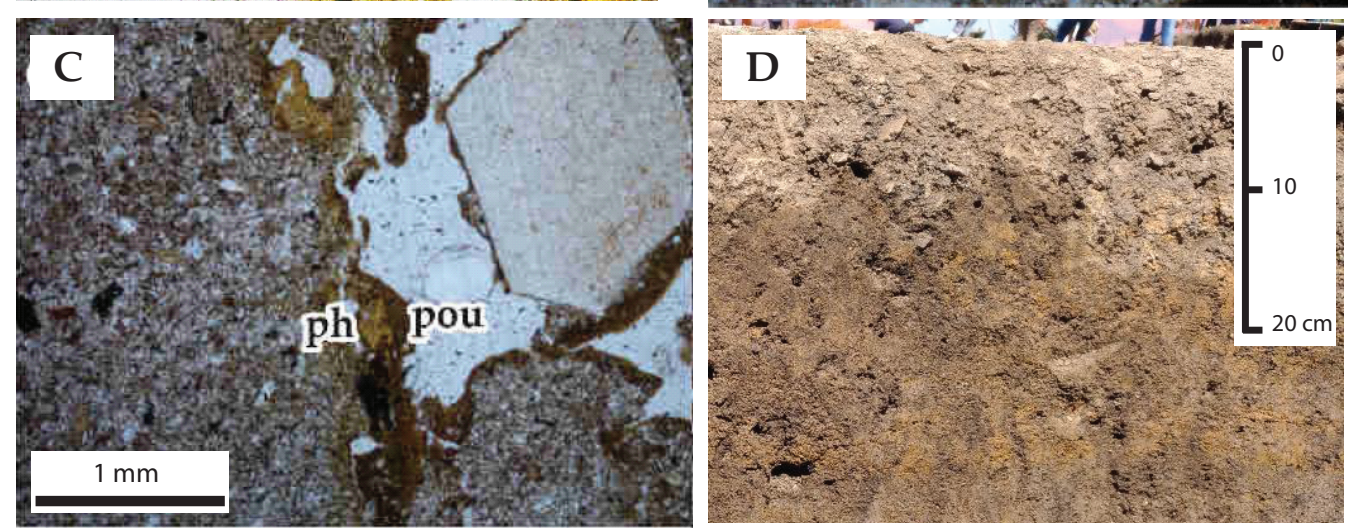
déduite par la découverte de micro-constituants dans des contextes de réutilisation. C'est le cas des glumes de graminée, issues du traitement des céréales, utilisées comme litières végétales sur les sols d'intérieurs du $X^{e}$ s., découverts à Gien (France) dans des bâtiments à vocation domestique [56], ou encore par la présence de très nombreuses déjections d'herbivores, riches en phytolithes, trouvées à Saint-Denis dans un contexte de zone humide en périphérie immédiate de l'agglomération du IX ${ }^{\mathrm{e}} \mathrm{s}$. [57].

Ces différents cas de figure montrent comment l'application d'une méthodologie couplant la micromorphologie, la géochimie, les données archéobotaniques, ouvre des perspectives intéressantes quant à l'interprétation de l'usage fait des espaces, leur qualification de «sale » ou «propre » et la pluralité des fonctions présentes.

\section{LA PÉDO-SÉDIMENTATION POST- ANTIQUE ET LES TERRES NOIRES}

Une des premières thématiques abordée par la géoarchéologie des contextes urbains, dès le début des années 1980, fut l'étude des terres noires [58]. Ces épaisses couches sombres, d'apparence homogène (fig. 4), mais qui peuvent contenir un abondant mobilier et des structures archéologiques plus diffuses, se retrouvent en effet de manière systématique dans les centres urbains anciens d'Europe. Leur interprétation a posé pendant longtemps des difficultés méthodologiques et elles ont trop souvent été enlevées à la

[56] BORDERIE et al. 2018.

[57] WYSS 2018.

[58] MACPHAIL 1981.
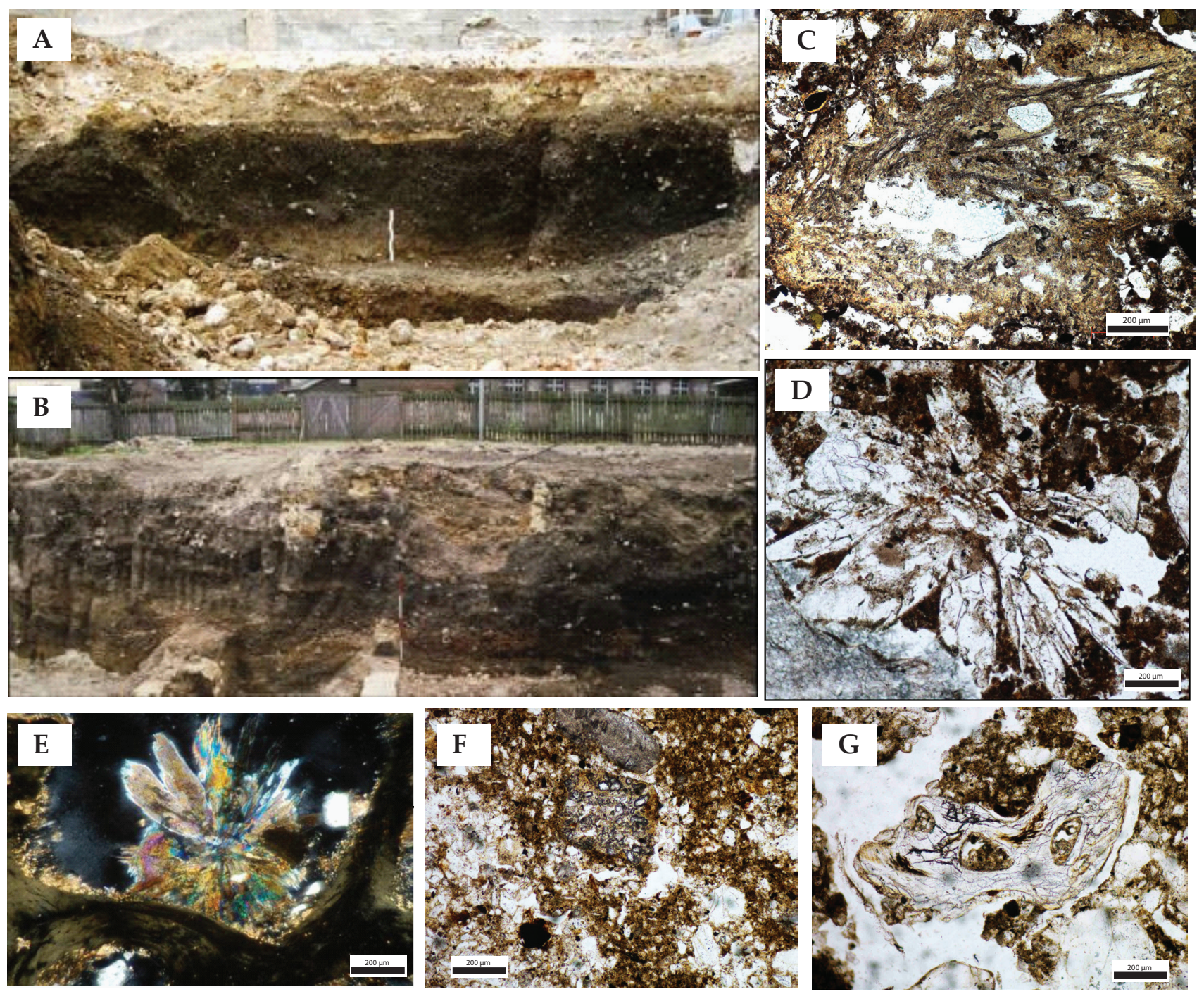

Figure 4

Terres noires. A : Évreux (cliché B. Guillot, Inrap), B : Noyon (cliché M. Talon) ; Microphotos (C - G) C : Excrément de grand herbivore, Noyon (LPnA); D : Cristal de gypse, Metz (LPnA); E : Vivianite dans un os, Noyon (LPA); F : Agrégat de silice fondue, Bayeux (LPnA); G : Fragment d'os chauffé, Bayeux (LPnA).

(D’après Borderie et al., 2012 ; Devos et al., 2011 ; Nicosia, 2018). 
pelleteuse, interprétées a priori comme synonymes de I'abandon des villes [59]. Pourtant, les terres noires constituent l'essentiel des archives de I'histoire de ces villes [60], et leur étude est reconnue aujourd'hui comme prioritaire. Depuis maintenant une trentaine d'année, I'association de fouilles fines à une approche combinant géochimie et micromorphologie, a montré la richesse des informations collectées [61]. Les occupations domestiques de la ville sont renseignées notamment par la richesse en micro-constituants liés aux activités culinaires, les occupations artisanales sont informées par la présence de matériaux chauffés à haute température (silice fondue, verre), la fréquentation est observable par le tassement des dépôts et la présence d'agrégats écrasés, roulés. Enfin, des

[59] GaLINIÉ 2004.

[60] CNRA 2002

[61] Borderie et al. 2014 ; CAMMAS 2004 ; Devos et al. 2011 ; MACPHAIL 1981 ; 1994 ; NiCOSIA 2018 ; Nicosia et al. 2017.

[62] Borderie 2016b; Borderie et al. 2012 ; Nicosia et al. 2017. horizons de surface très bien conservés peuvent être mis en évidence, par la présence de croûtes ou de phytolithes en connexion disposés sub-horizontalement. Ces activités prennent place sur un sol vivant, bioturbé à mesure de l'accrétion. Les terres noires sont des cumulosols anthropiques, qui recueillent les rejets d'activités urbaines très diversifiées et en aucun cas ne résultent d'un abandon, mais d'un usage multiple des espaces [62].

\section{MATÉRIAUX DE CONSTRUCTION}

Plus récemment, dans le prolongement des découvertes faites lors de l'étude des terres noires pour les contextes du début du Moyen Âge, les interrogations géoarchéologiques se sont portées sur les matériaux de construction. Si, au travers d'une archéologie du bâti surtout antique ou de la fin du Moyen Âge, la pierre, les mortiers et le bois sont des sujets d'étude récurrents, les matériaux en terre et en végétaux plus fins sont quasiment invisibles. Pourtant, I'analyse micromorphologique des sols d'intérieurs de bâtiments du premier Moyen Âge montre que, en France comme dans une grande part de l'Europe du
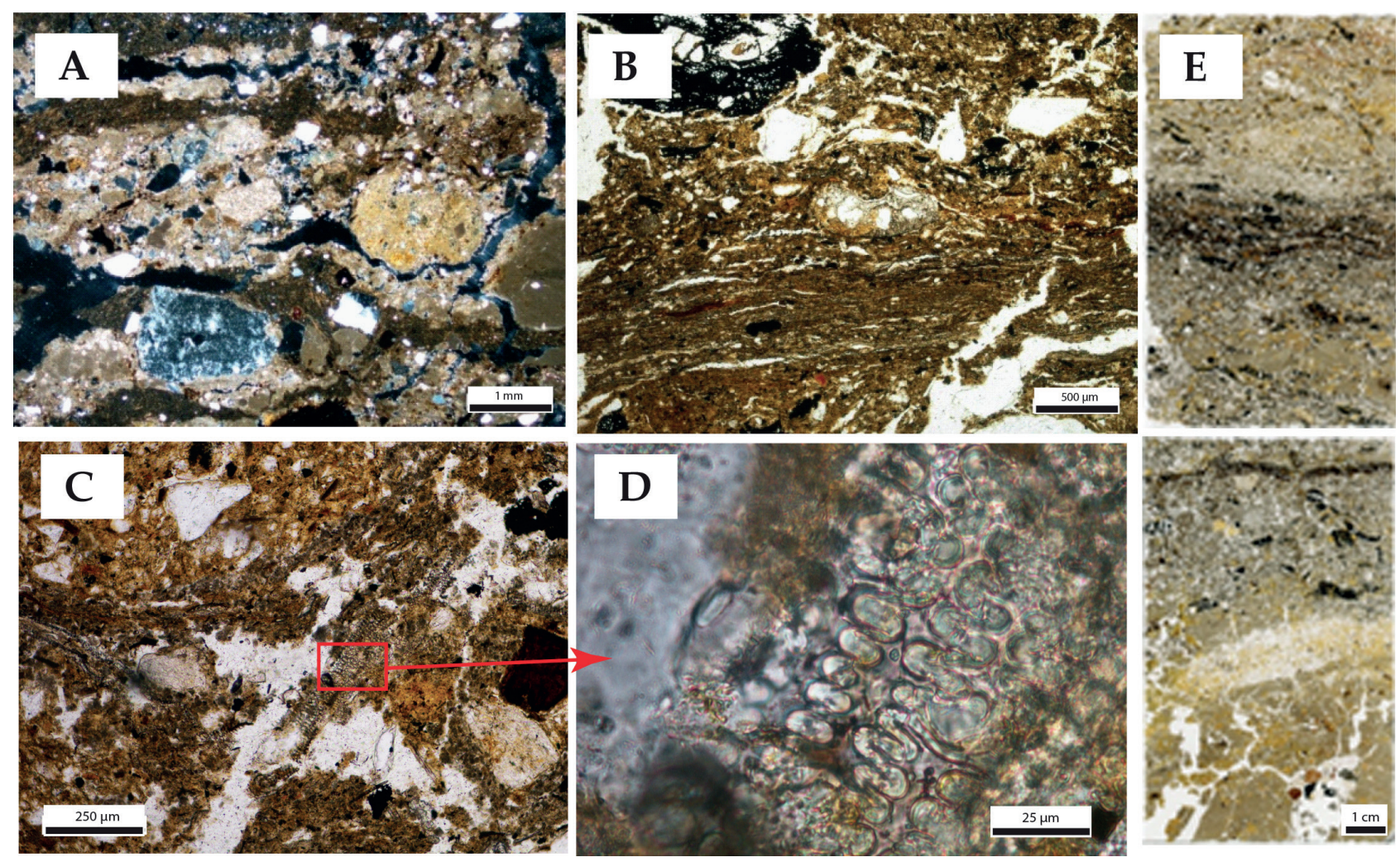

Figure 5

Sols d'intérieur de bâtiments. Microphotographies (A-D) : A : Alternance de sols argileux carbonatés et de couches de déchets (LPnA, Saint-Denis, France, XII<e S.) ; B : Sol végétal en graminées, surmontés par une couche de déchets contenant des cendres, de la silice fondue et des charbons fins (LPnA, Gien, France, $x^{e}$ s.) ; C : Sol végétal en graminées (LPnA, Gien, France, $x^{\mathrm{e}}$ s.) ; D : (zoom de C) phytolithe de céréale, probablement Triticum durum (LPnA, Gien, France, $X^{\mathrm{e}} \mathrm{s}$.) ; E : Scan de lames minces d'accumulations d'intérieur de maison (Ribe, Danemark, VIII ${ }^{\mathrm{S}}$.) (d'après Borderie et al., 2018 ; Croix et al., 2019 ; Wouters, 2016). 
Nord-Ouest, les architectures mobilisent des quantités considérables de limons, de sables et d'argile, de végétaux (fig. 5). Le cas de Gien et des jonchées de glumes de graminée a déjà été évoqué plus haut, ceux de Saint-Denis et de Noyon (France) sont tout aussi évocateurs. Pour ce dernier cas, la conservation en milieu humide de stratifications d'intérieur, mais aussi extérieures aux bâtiments, montre comment les végétaux constituent la majorité des accumulations dans les espaces occupés, où la réfection des surfaces est régulièrement alimentée par le dépôt de fines couches de limons ou de sables argileux [63]. À Ribe (Danemark), la fouille d'une parcelle des VIII $\mathrm{d}^{\mathrm{e}}-\mathrm{IX}^{\mathrm{e}} \mathrm{s}$. en 2018 a été couplée à une campagne systématique de prélèvements micromorphologiques. Il a pu être montré, ici aussi, la part importante des végétaux dans la constitution des stratifications internes aux bâtiments, par la construction des sols en végétaux (herbes, chaumes), recouverts de cendres et régulièrement rechapés d'argile [64]. La remobilisation de ces couches par des processus de bioturbation [65] ou de réaménagement des espaces [66] peut contribuer alors à la fabrication des terres noires. Les couches de préparation des sols d'occupation, régulièrement composées de limons tassés, en sont un autre exemple. Ces couches ont notamment été observées en Belgique, à Tongres [67], Bruxelles [68] et Ypres [69].

\section{PATRIMOINE ET CONSERVATION DES ARCHIVES}

\section{PÉDOGENÈSE ET PROCESSUS POST-ABANDON}

L'approche géoarchéologique, par définition, s'attache à comprendre les processus de formation des sites archéologiques [70], donc à l'évolution des pédo-stratifications. Les processus de recouvrement et d'érosion, le changement dans les activités et la fréquentation des lieux sont des problématiques régulièrement abordées, tout autant que le type de pédogenèse qui se met en place, puisque tous ont un impact sur la conservation des stratifications urbaines et l'interprétation qu'il est possible d'en faire. Dans le cas des agglomérations dites « abandonnées » de Silchester (Royaume-Uni) ou Gabies (Italie), le passage d'un usage du sol de type urbain à un usage agricole a été analysé. Dans le premier cas [71], pour l'insula III, il a été montré que les activités se limitaient progressivement à des actions de rejets de déchets culinaires, de fumier, puis de matériaux de construction altérés et dégradés, avant que ne se mette en place une pédogenèse, caractérisée par la structuration du sol en horizons différentiés. La préservation des espaces intérieurs et extérieurs de l'agglomération antique a donc été rendue possible par la rapidité des enfouissements. À Gabies [72], au contraire, les enfouissements sont très progressifs, du fait de la topographie du site, sur un versant. Les stratifications urbaines ont donc été recouvertes par des apports de colluvions issus de l'érosion de contextes urbains situés plus haut sur le versant. Un andosol s'est ensuite structuré, dont les agrégats pédologiques sont constitués pour une grande partie de dépôts archéologiques, siège d'une mise en culture dans sa partie supérieure. Dans les deux cas, les sols qui se structurent ont des matériaux parentaux d'origine anthropique, artificiels.

\section{CONSERVATION DE L'ENREGISTREMENT ARCHÉOLOGIQUE}

La densité des stratifications urbaines, et leur diversité, par l'analyse de multiples configurations d'enregistrements archéologiques permettent, pas à pas, de mieux comprendre les différentes modalités possibles quant à leur préservation. Ces paramètres sont un enjeu majeur pour la conservation patrimoniale, où les aménagements de sites peuvent modifier les conditions de milieux et, par là même, la qualité de l'enregistrement. Les recherches sur les terres noires ont été, à ce titre, particulièrement bénéfiques. Elles ont pu montrer comment les traces ténues d'occupations structurées de manière latente peuvent être perçues [73], mais aussi comment la bioturbation pouvait intervenir dans un environnement urbanisé et densément occupé, du fait de la nature même des productions sédimentaires anthropiques, riches en matières organiques [74]. Les indices indirects prennent alors tout leur sens lorsque la structuration macroscopique est peu lisible, telles ces pollutions organiques, aux métaux lourds, tels
[63] BORDERIE 2014

[64] CROIX et al. 2019.

[65] BORDERIE 2014.

[66] BORDERIE et al. 2018.

[67] WoUTERs et al. 2019.

[68] DEvos 2019.
[69] WOUTERS et al. 2018.

[70] SCHIFFER 1987.

[71] BANERJEA 2011.

[72] BORDERIE 2015.

[73] GÉBUS \& GAMA 2004

[74] Borderie 2014 ; CAMMAS 2004 ; MACPHAIL 1994. 
ces petits agrégats de sols d'intérieur de bâtiments, remobilisés, mais qui témoignent bien de la présence d'espaces bâtis [75].

Si le cadre urbain général de la conservation des stratifications, selon la nature des milieux, avait été théorisé dès les années 1980 [76], les documents d'évaluation du patrimoine archéologique des villes de France (Depavf) avaient rendu pratique I'utilisation de cette information, notamment dans les volumes consacrés à Soissons [77], où la profondeur estimée des destructions était figurée, et à Douai [78], où la possibilité d'une conservation de vestiges organiques était cartographiée. Quant aux risques fluviaux et littoraux en termes de conservation des vestiges, ce sont des paramètres non négligeables qui sont traités par la géoarchéologie [79].

\section{CONCLUSION}

\section{DES APPROCHES GÉOARCHÉOLOGIQUES}

Depuis son émergence dans les années 1980, la géoarchéologie des contextes urbains a donc connu une évolution des approches, de son objet d'étude et de sa pratique. De la géomorphologie et stratigraphie pratiquées dans la ville, I'on est passé progressivement à une géomorphologie anthropique de la ville, une « archéo-morphologie ». Cette discipline définit aujourd'hui pleinement ce qu'est la géoarchéologie des contextes urbains en considérant les espaces urbains comme des bassins de sédimentation anthropique autant que comme des artefacts, dont l'étude est nécessairement celle d'une co-construction société-milieux, pour la plus grande part artificiels. Depuis les années 1980, I'archéologie de sauvetage, puis l'archéologie préventive ont eu un rôle fondamental dans la mise en place de ces approches, lors des premières collaborations entre géographes, géologues et archéologues, dans la mise en pratique des méthodes, des transferts méthodologiques, jusqu'à la définition de problématiques interdisciplinaires, puis plus radicalement géoarchéologiques. Parmi les méthodes mobilisées, la micromorphologie a eu un rôle central et occupe désormais une place pivot dans l'analyse des stratifications et des sols urbains, au même titre que les approches spatiales et I'usage des SIG. Aujourd'hui, un des enjeux majeurs reste la permanence et la systématisation d'un suivi scientifique des sites urbains, par la présence d'une équipe locale [80].
Les cas de Lyon, mais aussi de Bruxelles sont à ce titre exemplaires et montrent tout le bénéfice gagné par le cumul et la mise en perspective de travaux conduits sur le long terme, de manière systématique [81]. Ce sont ces équipes qui peuvent, en outre, valoriser cette plus-value, scientifiquement, mais aussi auprès des concitoyens, des élus, des aménageurs, dans une relation vivante au sein de la cité.

\section{DE L'ÉTUDE DES SOCIO-ÉCOSYSTÈMES URBAINS ANCIENS À LA COMPRÉHENSION DES GÉO-SYSTÈMES À L'ANTHROPOCÈNE}

L'étude des contextes urbains nécessite donc d'aborder l'objet d'étude dans toute sa complexité, comme une co-construction des sociétés et d'un milieu le plus souvent artificiel. L'approche géoarchéologique de cet objet hybride s'affranchit de la notion de « remblais anthropiques » proposée à défaut par les cartes géologiques. Elle dépasse la notion de « matrix » avancée par A. Rosen [82] ou encore celle de «terres noires » [83], elle permet d'identifier et d'interpréter les dynamiques des socio-écosystèmes urbains qu'elle recouvre, d'aborder la diversité des activités humaines au-delà d'une vision fonctionnelle discontinue. Cette dynamique est celle de la gestion des flux de liquides et de solides, de déchets et de matériaux, la création d'anthropo-reliefs et d'anthropo-sols [84].

En définitive, la géoarchéologie des contextes urbains nous renseigne aujourd'hui autant sur les manières d'habiter un espace que sur le rapport au sol et au déchet que cela sous-tend : la gestion des espaces et des ressources, la gestion des risques et des héritages. Autant de problématiques qui, dans notre Anthropocène actuel hyper artificialisé pourraient fournir les clés d'une co-construction sur le temps long de ce qui fait l'urbain.

[75] Borderie et al. 2018 ; Devos et al. 2019.

[76] CARVER 1987.

[77] ROUSSELLE 2002.

[78] Demolon et al. 1990.

[79] CANUTI et al. 2000.

[80] BordERIE et al. 2018.

[81] Devos et al. 2019 ; FRANC 2016.

[82] ROSEN 1986.

[83] FONDRILLON 2009.

[84] DeSACHy 2008. 


\section{REMERCIEMENTS :}

Nous tenons à remercier les deux relecteurs anonymes pour leurs remarques très constructives, ainsi que Stéphane Gaillot, Michaël Wyss, Nicole Rodrigues, Hélène Noizet, Alexandra Legrand-Pineau, Mélinda Bizri, Richard Jonvel, Brigitte BoissavitCamus, Amélie Laurent, Hélène Dulauroy, Marie-Christine Lacroix, Bruno Desachy, Hélène Dulauroy-Lynch, Jean-Marc Fémolant, Sébastien Lefèbvre, Anne Gebhardt, Alain Queffelec, Pauline Dugas.

\section{BIBLIOGRAPHIE}

Allinne, Cécile, 2007, «Les villes romaines face aux inondations. La place des données archéologiques dans l'étude des risques fluviaux », Géomorphologie: relief, processus, environnement 13, p. 67-84.

Arlaud, Catherine, Burnouf, Joëlle, Bravard, Jean-Paul, Lurol, Jean-Marc \& Vérot-BourRéLy, Agnès, 1994, Lyon Saint-Jean : les fouilles de lîllot Tramassac. Service régional de l'archéologie, Lyon (Documents d'archéologie en Rhône-Alpes, 4. Série lyonnaise 10).

Arnaud-Fassetta, Gilles, Carcaud, Nathalie, Castanet, Cyril \& Salvador, Pierre-Gilles, 2010, « Fluviatile palaeoenvironments in archaeological context: Geographical position, methodological approach and global change - Hydrological risk issues », Quaternary International 216/1-2, p. 93-117, https://doi.org/10.1016/j.quaint.2009.03.009

Aubourg, Viviane \& Josset, Didier, 2000, « Le site du promontoire du château de Blois du VIII ${ }^{\mathrm{e}}$ au XI ${ }^{\mathrm{e}} \mathrm{S}$. (Loir-et-Cher). (Première partie : niveaux et structures) », Revue Archéologique du Centre de la France 39, p. 161-197.

AugrY, Stéphane \& BorderIE, Quentin, 2014, « Le jardin du cloître des carmélites de Metz (XVII ${ }^{\mathrm{e}-X V I I I^{\mathrm{e}}}$ siècles). Étude archéologique de l'aménagement d'un jardin en contexte urbain », Archéopages 37, p. 28-31. https://doi.org/10.4000/ archeopages.342

BaIze, Denis \& Girard, Michel-Claude, 2009, Référentiel pédologique 2008, Association Française pour l'Étude du Sol (Afes), Paris.

BANERJEA, Rowena Y., 2011, « Microscopic perspectives on the use of Period 3 MTRB 1/ERTB 1 », dans Michael Fulford \& Amanda Clarke, (éd.), Silchester: City in Transition. The Mid-Roman Occupation of Insula IX C. A.D. 125-250/300. A Report on Excavations Undertaken since 1997, London (Britannia Monograph Series), p. 63-95.

Barles, Sabine, Breysse, Denys, Guillerme, André \& Leyval, Corinne, 1999, Le sol urbain, Paris.

BeAU, Rémi \& LARRÈre, Catherine, 2018, Penser l'Anthropocène, Paris.

Bini, Monica, Fabiani, Fabio, Pappalardo, Marta \& Schuldenrein, Joseph, 2018, « Special issue of Geoarchaeology: Urban geoarchaeology in the Mediterranean Basin », Geoarchaeology: an International Journal 33, p. 3-12, https://doi.org/10.1002/gea.21674

BlieCK, Gilles \& Guiffray, Alain, 1994. «Genèse et évolution d'une place publique. L'exemple de Lille », dans Pierre

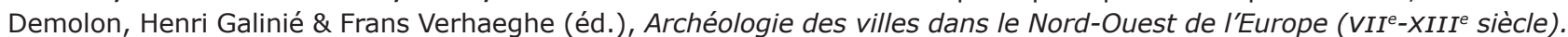
Actes du IVe Congrès International d'Archéologie Médiévale (Douai, 1991), Douai, p. 207-224.

Bonnet, Stéphane, JoRda, Christophe \& NrN, Nuria, 2010, « Nouvelles données sur la paléogéographie et le peuplement à Aix-en-Provence (Bouches-du-Rhône) : premiers résultats des approches géomorphologiques et géoarchéologiques », Quaternaire 21, p. 485-496.

Bonnet, Stéphane, Magnin, Frédéric, Nin, Nuria \& Dutour, Yves, 2017, Archéologie environnementale de la vallée de l'Arc de l'âge du Fer à l'Antiquité tardive. Rapport d'activité de la première année du PCR, Direction archéologie et muséum de la ville d'Aix-en-Provence, Aix-en-Provence.

Bonnet, Stéphane, Panneau, Marc \& Giroux, Hugues, 2018, « La modélisation au service de l'évaluation et de la caractérisation du sol urbain d'Aix-en-Provence », Les Nouvelles de l'Archéologie 153, p. 45-51, https://doi.org/10.4000/ nda.4865

BoRDERIE, Quentin, 2011a, « Géoarchéologie de I'urbain, retour sur la journée d'étude du 29 octobre 2009 », Les Nouvelles de l'Archéologie 123, p. 61-64, https://doi.org/10.4000/nda.1487

BorDerIe, Quentin, 2011b, L'espace urbain entre Antiquité et Moyen Âge, géoarchéologie des terres noires : études de cas, Thèse de doctorat, Archéologie et Environnement, Université de Paris 1 - Panthéon Sorbonne.

BORDERIE, Quentin, 2012, «Some quantitative aspect of thin section analysis for the archaeological research », dans Rosa M. Poch, Maria Casamitjana \& Michele L. Francis (éd.), Proceedings the $14^{\text {th }}$ International Working Meeting on Soil Micromorphology, Lleida 8-14 July 2012, Lleida.

BORDERIE, Quentin, 2014, «De la matrice sédimentaire aux pratiques socio-spatiales : deux stratifications de terres noires à Noyon (Oise) », dans Jeanne Brancier, Caroline Trémeaud \& Thibauld Valette (éd.), Des vestiges aux sociétés, regards croisés sur le passage des données archéologiques à la société sous-jacente, Paris, p. 61-64.

BorDerIE, Quentin, 2015, Gabies, expertise géoarchéologique, campagne 2014, Rapport non publié, Louvre, Paris. 
BorderIe, Quentin, 2016a, Chartres, Hôtel Montescot, étude géoarchéologique, stratigraphie et micromorphologie, Rapport non publié, Service Archéologique de la ville de Chartres, Chartres.

BorderIe, Quentin, 2016b, «Percevoir la complexité urbaine par l'étude de ses franges, les terres noires des IVe-XI siècles à Metz, Beauvais et Noyon », dans Claire Besson, Olivier Blin \& Bertrand Triboulot (éd.), Franges urbaines et confins territoriaux : la Gaule dans l'Empire, Bordeaux, p. 277-289.

Borderie, Quentin, Devos, Yannick, Nicosia, Cristiano, Cammas, Cécilia \& Macphail, Richard I., 2014, « Dark Earth in the geoarchaeological approach of urban contexts », dans Gilles Arnaud-Fassetta \& Nathalie Carcaud (éd.), French Geoarchaeology in the 21th century, Paris, p. 245-255.

Borderie, Quentin, Fondrillon, Mélanie, Nicosia, Cristiano, Devos, Yannick \& MacPhaIl, Richard I., 2014, «Bilan et nouveaux éclairages sur les terres noires : des processus complexes de stratification aux modalités d'occupation des espaces urbains », dans Xavier Rodier \& Elisabeth Lorans (éd.), Actes du 137e Congrès des Sociétés Historiques et Scientifiques, Université François Rabelais, Tours, 23-28 Avril 2012, Paris, p. 235-250.

Borderie, Quentin, Ball, Terry B., BanerJea, Y.R., BizRI, M., Lejault, C., Save, S. \& VAughan-Williams, A., 2018, « Early Middle Ages houses of Gien (France) from the inside: geoarchaeology and archaeobotany of 9th-11th C. floors », Environmental Archaeology, p. 1-19, https://doi.org/10.1080/14614103.2018.1534716

Borderie, Quentin, Bonnet, Stéphane, Bos, Teddy, Gaillot, Stéphane, Leconte, Pauline, Leroy, Eric, Meurisse-Fort, Murielle, Tronchère, Hervé, Germain-Vallée, Cécile \& Wuscher, Patrice, 2018, « Le paysage géo-archéologique de collectivité : milieu aride ou terre de culture? », Les Nouvelles de l'Archéologie 153, p. 29-38, https: / / doi.org/10.4000/nda.4749

Bravard, Jean-Paul, Vérot, Agnès, Burnouf, Joëlle, Amoros, Claude \& Jacquet, C., 1988, « Les fossés urbains en eau : proposition d'une analyse des conditions hydrodynamiques par une méthode morpho-écologique », dans Fouille, enregistrement et analyse des fossés et de leur comblement en milieu urbain, Actes de la Table Ronde organisée au Centre National d'Archéologie Urbaine à Tours les 23 et 24 avril 1988, Tours, p. 22-32.

Bravard, Jean-Paul., Burnouf, Joëlle \& VéRot, Agnès, 1989, « Géomorphologie et archéologie dans la région lyonnaise : questions et réponses d'un dialogue interdisciplinaire », Bulletin de la Société Préhistorique Française 86, p. 429-440. Burnouf, Joëlle, Carcaud, Nathalie, Cubizolle, Hervé, Garcin, Manuel, Trément, Frédéric \& Visset, Lionel, 2003, «Interactions sociétés/milieux dans le bassin versant de la Loire depuis le Tardiglaciaire jusqu'à la période industrielle », dans Tatiana Muxart, Franck-Dominique Vivien, Bruno Villalba \& Joëlle Burnouf, (éd.), Des milieux et des hommes : fragments d'histoires croisées, Paris, p. 65-77.

Burnouf, Joëlle \& LeVeau, Philippe, 2004, Fleuves et marais, une histoire au croisement de la nature et de la culture, Sociétés préindustrielles et milieux fluviaux, lacustres et palustres : pratiques sociales et hydrosystèmes, Paris.

BuTzer, Karl W., 1982, Archaeology as human ecology, Cambridge.

Butzer, Karl W., Miralles, Ismael \& Mateu, Juan F., 1983, « Urban geo-archaeology in medieval Alzira (Prov. Valencia, Spain) », Journal of Archaeological Science 10, p. 333-349, https://doi.org/10.1016/0305-4403(83)90071-7

CAmmas, Cécilia, 1994, «Approche micromorphologique de la stratigraphie urbaine à Lattes : premiers résultats. », Lattara 7, p. 181-202.

Cammas, Cécilia, 1999, «Dynamique pédo-sédimentaire urbaine : modes de construction et d'occupation à Lattes au IVe s. av. n. è. », Lattara 12, p. 211-227.

Cammas, Cécilia, 2003, « Microstratigraphie de la place 123, activités et rythmes de sédimentation au IVe s. 》, Lattara 16, p. 67-86.

CAmmAs, Cécilia, 2004, «Les «terres noires» urbaines du Nord de la France : première typologie pédo-sédimentaire », dans Laurent Verslype \& Raymond Brulet (éd.), Dark Earth - Terres Noires, Actes de la table ronde de Louvain-La-Neuve, Louvain-la-Neuve, p. 43-55.

Canuti, Paolo, Casagli, Nicola, Catani, Filippo \& Fantr, Ricardo, 2000, « Hydrogeological hazard and risk in archaeological sites: some case studies in Italy », Journal of Cultural Heritage 1, p. 117-125.

Carcaud, Nathalie, Arnaud-Fassetta, Gilles \& Evain, Caroline, 2019, Villes et rivières de France, Paris.

CARVER, Martin O. H., 1987, «The nature of urban deposits », dans John Schofield \& Roger Leech (éd.), Urban Archaeology in Britain (CBA Research report 61), London, p. 9-26.

Cheverry, Claude \& Gascuel, Chantal, 2009, Sous les pavés la terre, Montreuil.

CNRA, 2002, « La recherche archéologique en France. Bilan 1995-1999 du Conseil national de la recherche archéologique », Les nouvelles de l'archéologie 88.

CoHEN, Nathalie \& WRAGG, Eliott, 2017, «The River's Tale »: Archaeology on the Thames Foreshore in Greater London, London.

Cook, Samantha R., Banerjea, Rowena Y., Marshall, L.-J., Fulford, M., Clarke, A. \& Van Zwieten, C., 2010, «Concentration of copper, zinc and lead as indicator of hearth usage at the Roman town of Calleva Atrebatum (Silchester, Hampshire, UK) ». Journal of Archaeological Science 37, p. 871-879, https://doi.org/10.1016/j.jas.2009.11.017

Cook, Samantha R., Clarke, Amanda, Fulford, Michael \& Voss, Jochen, 2014, « Characterising the use of urban space: a geochemical case study from Calleva Atrebatum (Silchester, Hampshire, UK) Insula IX during the late first/early second century AD », Journal of Archaeological Science 50, p. 108-116, https://doi.org/10.1016/j.jas.2014.07.003

Coussor, Céline, 2008, Étude géoarchéologique du site C219, Rapport non publié, Service Archéologique de la ville de Chartres.

Croix, Sarah, Deckers, Pieterjan, Feveile, Claus, Knudsen, M., SkytTe QuistgaArd, S., Sindbak, S.M. \& Wouters, Barbora, 2019, «Single Context, Metacontext, and High Definition Archaeology: Integrating New Standards of Stratigraphic Excavation and Recording », Journal of Archaeological Method and Theory 26, p. 1-42, https://doi.org/10.1007/ s10816-019-09417-x 
Davidson, Donald A., 1973, « Particle size and phosphate analysis: evidence for the evolution of a tell », Archaeometry 15, p. 143-152.

Davidson, Donald A., 1976, « Processes of tell formation and erosion », dans Donald A. Davidson \& Myra L. Shackley (éd.), Geoarchaeology: Earth Science and the Past, London, p. 255-266.

Davidson, Donald A., Dercon, Gerd, Stewart, Mairi \& Watson, Fiona, 2006, « The legacy of past urban waste disposal on local soils », Journal of Archaeological Science 33, p. 778-783, https://doi.org/10.1016/j.jas.2005.10.017

Demolon, Pierre, Lours, Etienne \& Louis-VAubanCe, Murielle, 1990, Document d'évaluation du patrimoine archéologique des villes de France : Douai, Tours.

DesaChy, Bruno, 2008, De la formalisation du traitement des données stratigraphiques en archéologie de terrain, Thèse de doctorat, Université de Paris 1 Panthéon-Sorbonne.

Descola, Philippe, 2005, Par-delà nature et culture, Paris.

Devos, Yannick, 2019, Dark Earth in Brussels (Belgium). A geoarchaeological study, Thèse de doctorat, Vrije Universiteit Brussel.

Devos, Yannick, Vrydaghs, Luc, Degraeve, Ann \& Modrie, Sylvianne, 2011, « Unravelling urban stratigraphy. The study of Brussels' (Belgium) Dark Earth. An archaeopedological perpective », Medieval and Modern Matters 2, p. 51-76, https://doi.org/10.1484/J.MMM.1.102776

Devos, Yannick, Nicosia, Cristiano, Vrydaghs, Luc, Speleers, L., Van der Valk, J., Marinova, Elena, Claes, Britt., Albert, Rosa Maria, Esteban, Irene, Ball, Terry B., Court-Picon, Mona \& Degraeve, Ann, 2017a, «An integrated study of Dark Earth from the alluvial valley of the Senne river (Brussels, Belgium) », Quaternary International 460, p. 175-197, https://doi.org/10.1016/j.quaint.2016.06.025

Devos, Yannick, Van Neer, Wim, Marinova, Elena \& Degraeve, Ann, 2017b, « Editorial: Proceedings of the Conference on the Environmental Archaeology of European Cities (CEAEC) », Quaternary International 460, p. 1-2, https:// doi.org/10.1016/j.quaint.2017.06.012

Devos, Yannick, Nicosia, Cristiano, Vrydaghs, Luc, Langohr, Roger \& Marinova, Elena, 2017c, « L'évolution du paysage bruxellois, un regard archéologique », Bruxelles Patrimoines 23-34, p. 8-17.

Devos, Yannick, Nicosia, Cristiano \& Wouters, Barbora, 2019, « Urban geoarchaeology in Belgium: Experiences and innovations », Geoarchaeology: an international Journal, p. 1-15, https://doi.org/10.1002/gea.21755

FondRILLON, Mélanie, 2009, «À propos des recherches sur les terres noires urbaines : dépasser le concept d'attente », Archéologie Médiévale 39, p. 1-16.

GaLINIÉ, Henri, 2000, Ville, espace urbain et archéologie, Tours.

GALINIÉ, Henri, 2004, «L'expression «terres noires», un concept d'attente », dans Laurent Verslype \& Raymond Brulet (éd.), Dark Earth - Terres Noires, Actes de la Table Ronde de Louvain-La-Neuve, Louvain-la-Neuve, p. 1-11.

GARMY, Pierre, 2009, Villes, réseaux et systèmes de villes : contribution de l'archéologie, thèse d'Habilitation à diriger les Recherches, Université de Paris 1 Panthéon-Sorbonne.

GÉ, Thierry, Courty, Marie-Agnès, Matthews, Wendy \& WATtez, Julia, 1993, « Sedimentary formation processes of occupation surfaces », dans Paul Goldberg, David T. Nash \& Michael Petraglia (éd.), Formation Processes in Archaeological Context, Madison, Wisconsin (Monographs in world archaeology 17), p. 149-163.

GÉBus, Laurent \& GAMA, Franck, 2004, « Le quartier de la Pierre-Hardie et l'îlot Turmel dans la ville de Metz en Lorraine : deux exemples d'études archéologiques des couches sombres attribuées aux IV ${ }^{-}-\mathrm{XII}^{\mathrm{e}}$ siècles », dans Laurent Verslype \& Raymond Brulet (éd.), Dark Earth - Terres Noires, Actes de la Table Ronde de Louvain-La-Neuve, Louvain-la-Neuve, p. $135-144$. GelAUDE, Frank, 2019. «De ontwikkeling en groei van de rivierhaven Gent tussen 1100 en 1300 (O.-VI.) 》, Archaeologia Medievalis, Chronique, Kroniek, Chronik 42, p. 66.

Goiran, Jean-Philippe, Tronchère, Hervé, Salomon, Ferréol, Carbonel, Pierre, Djerbi, Hatem \& Ognard, Carole, 2010, «Palaeoenvironmental reconstruction of the ancient harbors of Rome: Claudius and Trajan's marine harbors on the Tiber delta », Quaternary International 216/1-2, p. 3-13, https://doi.org/10.1016/j.quaint.2009.10.030.

Goiran, Jean-Philippe, Salomon, Ferréol, Mazzini, Ilaria, Bravard, Jean-Paul, Pleuger, Elisa, Vittori, Cécile, BoEtTo, Guilia, et al., 2014, « Geoarchaeology confirms location of the ancient harbour basin of Ostia (Italy) », Journal of Archaeological Science 41, p. 389-398, https://doi.org/10.1016/j.jas.2013.08.019

GoldING, Kirsti A., 2008, The effect of waste disposal on soils in and around historic small towns, PhD Thesis, University of Stirling.

HALl, Allan R. \& KenwaRd, Harry K. (éd.), 1982. Environmental archaeology in the urban contexts, CBA Research Report, Council for British Archaeology, London.

Hrgelin, Mathias, 2016, «L'agglomération romaine d'Horbourg-Wihr. Carte archéologique et synthèse thématique », Cahiers Alsaciens d'Archéologie d'Art et d'Histoire, 59, p. 81-102.

LAURENT, Amélie, 2007, Évaluation du potentiel archéologique du sol en milieu urbain, Thèse de doctorat, Université François Rabelais, Tours.

LAURENT, Amélie \& Fondrillon, Mélanie, 2010, « Mesurer la ville par l'évaluation et la caractérisation du sol urbain : I'exemple de Tours ». Revue Archéologique du Centre de la France 49, p. 307-343.

Le Roux, Gaël, Véron, Alain \& MoRHANGe, Christophe, 2005, « Lead pollution in the ancient harbours of Marseille », Méditerranée 104, p. 31-35.

Lebret, Patrick, Campy, Michel, Coutard, Jean-Pierre, Fourniguet, Jackie, Isambert Michel, LAutridou, Jean-Paul, Laville, Pierre, MaCaIre, Jean-Jacques, Menillet, François \& MeYer, Robert, 1993, « Cartographie des formations superficielles. Réactualisation des principes de représentation a 1/50000 », Géologie Française 4, p. 39-54. 
LEPETIT, Bernard, 1996, «La ville : cadre, objet, sujet. Vingt ans de recherches françaises en histoire urbaine », Enquête. Anthropologie, Histoire, Sociologie 4, https://doi.org/10.4000/enquete.663

LeVY, Jacques \& Lussault, Michel, 2003, Dictionnaire de la Géographie et de l'espace des sociétés, Paris.

MACPHAIL, Richard I., 1981, « Soil and botanical studies of the "Dark Earth" », dans Martin Jones \& G. W. Dimbleby (éd.), The Environment of Man: The Iron Age to the Anglo-Saxon Period, Oxford (BAR British Series 87), p. 309-331.

MACPHAIL, Richard I., 1983, « The micromorphology of dark earth from Gloucester, London and Norwich: an analysis of urban anthropogenic deposits from the Late Roman to Early Medieval periods in England », dans P. Bullock \& C. P. Murphy (éd.), Soil Micromorphology, vol. 1 Techniques and Applications, Rothamsted, p. 245-252.

MACPHAIL, Richard I., 1994, « The Re-working of urban stratigraphy by human and natural processes », dans Allan R. Hall \& Harry K. Kenward, (éd.), Urban-Rural Connexion: Perspectives from Environmental Archaeology, Oxford (Symposia of the Association for Environmental Archaeology 12), p. 13-44.

Magny, Michel, 2019, Aux racines de l'Anthropocène, une crise écologique reflet d'une crise de l'homme, Lormont.

Marriner, Nick, Morhange, Christophe \& Goiran, Jean-Philippe, 2010, « Coastal and ancient harbour geoarchaeology: a short review », Geology Today 26, p. 21-27.

Matthews, Wendy, 1992, The micromorphology of occupational sequences and the use of space in a Sumerian city, PhD thesis, University of Cambridge.

Matthews, Wendy, French, Charles A.I., Lawrence, T., Cutler, D.F. \& Jones, M.K., 1997, « Microstratigraphic traces of site formation processes and human activities », World Archaeology 29, p. 281-308.

Matthews, Wendy, Postgate, Nicholas, Paynes, S., Charles, M. P. \& Dobney, K., 1995, « The Imprint of Living in an Early Mesopotamian City: questions and answers », dans Rosemary-Margaret Luff \& P. Rowley-Conway (éd.), Whither Environmental Archaeology?, Oxford (Oxbow Monograph 38), p. 171-212.

MonrN, Michèle, 2005, « Le murus gallicus de Lyon (Rhône) », Bulletin de l'Association Française pour l'étude l'âge Fer 33, p. 67-71.

Morhange, Christophe, 2001, Mobilité littorale de quelques sites portuaires antiques de Méditerranée : Marseille, Pouzzoles, Cumes, Kition et Sidon, Thèse de doctorat, Université de Provence - Aix-Marseille I, https://tel.archives-ouvertes. fr/tel-00269281

Morhange, Christophe, Provensal, Mireille, Hesnard, A., Bouiron, Marc, Bourcier, Michel, Carbonel, Pierre \& Weydert, Pierre, 1996, « Morphogenèse et impacts anthropiques sur les rives du Lacydon à Marseille (6000 av. J.-C. - 500 ap. J.-C.) », Annales de Géographie 105, p. 32-46.

MuXart, Tatiana, Vivien, Franck-Dominique, Villalba, Bruno \& Burnouf, Joëlle, 2003, Des milieux et des hommes : fragments d'histoires croisées, Paris.

Nicosia, Cristiano, 2018, Geoarcheologia delle stratificazioni urbane postclassiche, Antenor Quaderni 45, Roma.

Nicosia, Cristiano, Devos, Yannick \& MacPhaIl, Richard I., 2017, « European Dark Earth », dans Cristiano Nicosia \& George Stoops (éd.), Archaeological Soil and Sediment Micromorphology, Oxford, p. 331-343.

NiN, Nuria, 2002, «L'artisanat à Aix-en-Provence durant l'Antiquité », dans Jean-Claude Béal \& Jean-Claude Goyon (éd.), Les artisans dans la ville antique, Lyon, p. 235-250.

NoIzet, Hélène, Boves, Boris \& CostA, Laurent, 2013, Paris de parcelles en pixels, Paris.

Ollive, Vincent, Petit, Christophe, Garcia, Jean-Pierre \& RÉdDÉ, Michel, 2006, « Rhine flood deposits recorded in the Gallo-Roman site of $\mathbb{E}$ denburg (Haut-Rhin, France) ». Quaternary International 150, p. 28-40.

Paquot, Thierry, Lussault, Michel \& Body-Gendrot, Sophie, 2000, La ville et l'urbain : l'état des savoirs, Paris. Peiry, Jean-Luc \& Féougrer, Cyril, 1997, «À propos de la fondation de Grenoble : un réexamen de l'hypothèse d'une installation sur un môle alluvial », dans Jean-Paul Bravard \& Michel Prestreau, (éd.), Dynamique du paysage : entretiens de géoarchéologie, Actes de la Table Ronde des 17 et 18 novembre 1995, Lyon (Document d'Archéologie en Rhône Alpes 15), p. 169-176.

Pons-Branchu, Edwige, Roy-Barman, Matthieu, Jean-Soro, Liliane, Guillerme, André, Branchu, Philippe, Fernandez, Matthieu, Dumont, Emmanuel, Douville, Eric, Michelot, Jean-Luc \& Phillips, Andy, 2017, «Urbanization impact on sulfur content of groundwater revealed by the study of urban speleothems: Case study in Paris, France », Science of the Total Environment 579, p. 124-132.

Rosen, Arlen M., 1986, Cities of clay, the geoarchaeology of tells, Chicago.

Roth-Zehner, Muriel, 2018, Horbourg-Wihr (Haut-Rhin) : 50 Grand'Rue : Fouille d'un quartier antique au centre de Horbourg-Wihr, Rapport de fouilles programmées, Sélestat.

Rousselle, Dominique, 2002, Document d'évaluation du patrimoine archéologique des villes de France : Soissons, Tours. SALomon, Ferréol, 2013. Géoarchéologie du delta du Tibre : Évolution géomorphologique holocène et contraintes hydrosédimentaires dans le système Ostie - Portus, thèse de doctorat, Université de Lyon 2.

Salomon, Ferréol, Goiran, Jean-Philippe, Noirot, Brice, Pleuger, Elise, Bukowiecki, Evelyne, Mazzini, Ilaria \& CARBONel, Pierre, 2018, « Geoarchaeology of the Roman port-city of Ostia: Fluvio-coastal mobility, urban development and resilience », Earth-Science Review 177, p. 265-283, https://doi.org/10.1016/j.earscirev.2017.10.003

SCHIFFER, Michael B., 1987, Formation processes of archaeological records, Albuquerque.

Schwren, Jean-Jacques (éd.), 1991, Tramway de l'agglomération strasbourgeoise. Étude d'impact archéologique, Strasbourg.

Schwien, Jean-Jacques, Schnerder, Nathalie \& WATton, Marie-Dominique, 1998, « Le site naturel de Strasbourg et ses aménagements hydrographiques de I'Antiquité à l'époque moderne », Archéologie Médiévale 28, p. 33-70.

Sear, David A., Bacon, S.R., Murdock, A., Doneghan, G., Baggaley, P., Serra, C. \& LeBas, T.P., 2011, «Cartographic, Geophysical and Diver Surveys of the Medieval Town Site at Dunwich, Suffolk, England », International Journal of Nautical Archaeology 40, p. 113-132. 
Thion, Pierre, Faye, Olivier, Georges-Leroy, Murielle, Dreidemy, Christophe \& Périchon, Denis, 2003, «Les fortifications gauloises de Metz (Moselle) », Annales de l'Est, La fortification en Lorraine : de l'enceinte gauloise de Metz à la ligne Maginot, Nancy, p. 7-34.

Timmermans, Julie, Devos, Yannick, Ghesquière, Valérie, De Cupere, Bea, Deforce, Koen, Speleers, Lien, VRYdAghS, Luc \& VAN Bellingen, Stephan, 2020, «Brucity/parking 58 : Un ancien quai de Senne (XVe siècle) (RBC/ BHG) », Archaeologia Medievalis Chronique-Kroniek-Chronik 43, p. 80-81.

Vella, Marc-Antoine, Ghilardi, Matthieu, Diouf, Ousmane, Parisot, Jean-Claude, Hermitte, Daniel, Provansal, Mireille, Fleury, Jules, Dussouillez, Philippe, Delanghe-Sabatier, Doriane \& Demory, François, 2013, « Géoarchéologie du Rhône dans le secteur du pont Saint-Bénézet (Avignon, Provence, France) au cours de la seconde moitié du deuxième millénaire apr. J.-C.: étude croisée de géographie historique et des paléoenvironnements ». Géomorphologie Relief Processus Environments 19, p. 287-310.

Vurllemin Adrien, 2016, «La Cour de Lorraine à Mulhouse, du Haut-Empire à la Seconde Guerre Mondiale », Annuaire historique de la Ville de Mulhouse 27, p. 147-163.

Weber, Max, 1921, Die Stadt, (trad. P. Fritsch - La Ville, Paris, 1982).

Wickham, Chris I., 2005, Framing the Early Middle Ages, Oxford.

WouTERS, Barbora, 2016, Geoarchaeological and micromorphological approaches to the formation and biographies of Early Medieval towns in Northwest Europe, Thèse de doctorat, Vrije Universiteit Brussel/University of Aberdeen.

Wouters, Barbora, Devos, Yannick, Bartholomieux, Bart \& Bradt, Tomas, 2018, « Towns on the edge: Urban geoarchaeology of medieval Ieper (Colaertplein) », dans $4^{\text {th }}$ EAA Annual Meeting (Barcelona, 2018). Abstract Book. Barcelona, p. 566.

Wouters, Barbora, Devos, Yannick, Milek, Karen, VRydaghs, Luc, Bartholomieux, Bart, Tys, Dries, Moolhuizen, Cornelie \& VAN AsCh, Nelleke, 2017, « Medieval markets: A soil micromorphological and archaeobotanical study of the urban stratigraphy of Lier (Belgium) », Quaternary International 460, p. 48-64.

Wouters, Barbora, Devos, Yannick, Vrydaghs, Luc, Ball, Terry B., De Winter, Natasja \& Reygel, Patrick, 2019, «An integrated micromorphological and phytolith study of urban soils and sediments from the Gallo-Roman town Atuatuca Tungrorum (Tongeren, Belgium) », Geoarchaeology 34, p. 448-466.

Wyss, M., 2018, «Plus d'un millénaire de techniques hydrauliques dans le castellum de Saint-Denis », dans Actes des journées archéologies d'île-de-France, novembre 2017, Paris. 